\title{
HOMOGENIZATION OF THE SYSTEM OF HIGH-CONTRAST MAXWELL EQUATIONS
}

\author{
KIRILL CHEREDNICHENKO AND SHANE COOPER
}

\section{To Vladimir Gilelevich Maz'ya on the occasion of his 75th anniversary}

Abstract. We study the system of Maxwell equations for a periodic composite dielectric medium with components whose dielectric permittivities $\epsilon$ have a high degree of contrast between each other. We assume that the ratio between the permittivities of the components with low and high values of $\epsilon$ is of the order $\eta^{2}$, where $\eta>0$ is the period of the medium. We determine the asymptotic behaviour of the electromagnetic response of such a medium in the "homogenization limit", as $\eta \rightarrow 0$, and derive the limit system of Maxwell equations in $\mathbb{R}^{3}$. Our results extend a number of conclusions of a paper by Zhikov [On gaps in the spectrum of some divergent elliptic operators with periodic coefficients. St. Petersburg Math. J. 16(5) (2004), 719-773] to the case of the full system of Maxwell equations.

$\S 1$. Introduction. In the present article we carry out the asymptotic analysis of an operator family that has been coming, since the mid 1990s, to the fore of attention by researchers in a number of disciplines, in relation to wave propagation through periodic composite materials. The family in question has been proposed as a mathematical model of the "photonic crystal", a periodic material that prohibits propagation of waves in specified frequency ranges [15], which correspond to gaps (or "lacunae") in the spectra of the operators involved. This property of a photonic crystal is impossible in usual periodic media at high frequencies, as was recently proved in [23] for the Schrödinger operator of quantum mechanics. In the early days of photonic crystals, it was also suggested that the above band-gap effect can be exploited in the design of "photonic crystal fibres", which ensure that waves of a given frequency propagate in a specific direction without much interference from the outside [25]. A number of mathematical works followed, including [12, 13, 16, 28, 29], which provided an analytical framework for these physical phenomena in the setting of a scalar wave equation. The main thrust of the related analysis is on the spectral properties of a family of self-adjoint differential operators of second order with periodic coefficients. However, in contrast to the "classical" case of a

Received 13 May 2014, published online 27 February 2015.

MSC (2010): 35J15, 35J70, 47F05 (primary).

(c) 2015 University College London. This article is distributed with Open Access under the terms of the Creative Commons Attribution License (http://creativecommons.org/licenses/by/4.0/), which permits unrestricted reuse, distribution, and reproduction in any medium, provided that the original work is properly cited. 
periodic composite medium (see e.g. $[3, \mathbf{1 4}, \mathbf{2 1}]$ ), where the analysis of the fibre decomposition [19] leads to a homogeneous effective medium in the limit of vanishing period, in the case of a photonic crystal one deals with operator families whose ellipticity constants go to zero along with the period in the coefficients. These are often referred to as high-contrast problems, on account of the contrast between different material components in a composite, which increases as the period becomes smaller.

The subsequent progress in the mathematical analysis of photonic crystals has proceeded in several directions. On the one hand, new classes of structures have been considered, e.g. periodic arrangements of "thin" structures (see [24] and references therein), shedding a new light on the earlier achievements of asymptotic analysis of networks and junctions (see e.g. [18]). This activity is also related to the study of spectral properties of "quantum graphs", i.e. structures of "zero thickness" (see e.g. [4] and references therein). On the other hand, the analysis of the full vectorial (i.e. three-dimensional) problems in the context of linearized elasticity or electromagnetism, which seems to be a natural objective from the applied point of view, has been pursued. This presents an additional challenge compared to the scalar case, due to the more complicated geometric structure of the operators involved and the lack of certain technical tools for systems of partial differential equations, such as e.g. the maximum principle.

From the perspective of the mathematical theory of homogenization, nonuniformly elliptic operator families are often referred to as "non-standard", due to the fact that sequences of elements of the operator domains that have uniformly bounded energies are not necessarily compact with respect to the standard energy norm. It implies, in particular, that the usual "one-scale" $L^{2}$ topology does not suffice for obtaining a homogenized limit whose energy is the limit of the energies of the original sequences ("energy identity"), and one has to resort to a more advanced multiscale $L^{2}$-topology on a product of the "slow" (macroscopic) and "fast" (microscopic) domains of the independent variable. Another consequence of this lack of compactness is the two-scale structure of the limit operator; see e.g. [28]. Several analytical tools, which have been developed to study homogenization problems, allow us to treat this multiscale aspect of high-contrast problems, including the method of two-scale convergence [2, 22], the method of periodic unfolding [8], the method of multiscale asymptotic expansions $[\mathbf{5}, \mathbf{2 6}]$ and the method of resolvent asymptotics in the Bloch space (see [6]; see also [17], where a general approach to degenerate problems is discussed, based on the generalized Weyl decomposition). Arguably, any of these techniques can be employed for the analysis of the full system of Maxwell equations, which is of interest to us in the present work. Based on our previous experience (see e.g. $[6,7,9]$ ), we adopt the method of two-scale convergence in combination with the analysis of the "space of microscopic oscillations" for the Maxwell operator, which in the general form was introduced in [17].

The structure of the present paper is as follows. In $\$ 2$ we outline the problem at hand and formulate the main result of the paper. In $\$ 3$ we prove several statements used in the subsequent analysis of equations (5). These concern the 
structure of the null set of the curl-operator on $Q_{1}$ and the well-posedness of the associated unit-cell problems. Section 4 is devoted to the proof of the main result, which consists of several stages, namely: the proof of two-scale compactness of solution sequences $u^{\eta}$, and their gradients, for equations (5) with right-hand sides $f^{\eta}$ that are uniformly bounded in $\left[L^{2}\left(\mathbb{R}^{3}\right)\right]^{3}$, as well as the "structural relations" between limits of sequences and their scaled curls (Lemma 4.1); the proof of "macroscopic regularity" of the two-scale limit of the solutions $u^{\eta}$ (Proposition 4.3); the derivation of the limit system (6)-(8) in the main theorem (Theorem 2.1); ending with the characterization of $A^{\text {hom }}$ (Lemma 4.4) and the study of the solution set of (6)-(8).

§2. Problem formulation and main result. A time-harmonic electromagnetic wave $E, H$ propagating with frequency $\omega$ through a three-dimensional dielectric composite is governed by the system of Maxwell equations in $\mathbb{R}^{3}$ :

$$
\begin{aligned}
& \operatorname{curl} E=\mathrm{i} \omega \mu H, \\
& \operatorname{curl} H=-\mathrm{i} \omega \epsilon E
\end{aligned}
$$

with

$$
\operatorname{div}(\epsilon E)=0 \quad \text { and } \quad \operatorname{div}(\mu H)=0 .
$$

Here $\mu$ and $\epsilon$ are the magnetic permeability and electric permittivity of the dielectric medium. This article considers the class of problems described by $\mu=1$ and $\epsilon(x)=\epsilon_{\eta}(x)=\epsilon_{1}(x / \eta)+\eta^{-2} \epsilon_{0}(x / \eta), \eta \in\left(0, \frac{1}{2}\right)$, where $\epsilon_{0}, \epsilon_{1}$ are periodic scalar functions with respect to $Q=[0,1)^{3}$ and are such that

$$
\operatorname{supp}\left(\epsilon_{i}\right)=\overline{Q_{i}}, \quad 0<v \leqslant\left(\left.\epsilon_{i}\right|_{Q_{i}}\right)^{-1} \leqslant v^{-1}, \quad i=0,1,
$$

where $Q_{0}$ is an open $C^{2}$-set compactly contained in $Q$ and such that $Q_{1}:=$ $Q \backslash \overline{Q_{0}}$ is simply connected. Important questions in applications concern the sets $S_{\eta}:=\left\{\omega^{2}: \exists\right.$ a non-trivial (quasiperiodic) solution $E, H$ to (1)-(3) $\}, \eta \in\left(0, \frac{1}{2}\right)$,

and their limit behaviour as $\eta \rightarrow 0$. By a straightforward manipulation, it is seen that if $\lambda=\omega^{2} \in S_{\eta}$, then the corresponding magnetic component $H$ solves the "reduced" system

$$
\operatorname{curl}\left(\epsilon_{\eta}^{-1} \operatorname{curl} H\right)=\lambda H, \quad \operatorname{div} H=0,
$$

where $\epsilon_{0}^{-1}, \epsilon_{1}^{-1}$ are the inverses of $\epsilon_{0}, \epsilon_{1}$ on their support and zero elsewhere. In other words, we find that the set $S_{\eta}$ is the spectrum $\sigma\left(A_{\eta}\right)$ of the self-adjoint operator $A_{\eta}$ associated with the problem (4). With the aim of analysing the behaviour of $S_{\eta}$ in a future work, we focus in this article on the convergence of the family of resolvent operators $\left(A_{\eta}+I\right)^{-1}$ with respect to the two-scale topology; see [2, 22, 28]. Namely, for a given family of divergence-free functions $f^{\eta}$ two-scale converging to $f$, we shall study the two-scale convergence of the 
family $u^{\eta}$ of (weak) solutions to

$$
\operatorname{curl}\left(\epsilon_{\eta}^{-1} \operatorname{curl} u^{\eta}\right)+u^{\eta}=f^{\eta}, \quad \operatorname{div} u^{\eta}=0 \quad \text { in } \mathbb{R}^{3} .
$$

We now present our main result.

THEOREM 2.1. Suppose that a sequence $f^{\eta} \in\left[L^{2}\left(\mathbb{R}^{3}\right)\right]_{\text {sol }}^{3}$ weakly (strongly) two-scale converges to $f \in\left[L^{2}\left(\mathbb{R}^{3} \times Q\right)\right]^{3}$ as $\eta \rightarrow 0$. Then the sequence of solutions $u^{\eta} \in\left[H^{1}\left(\mathbb{R}^{3}\right)\right]^{3}$ to the equations (5) weakly (strongly) two-scale converges to the function $u^{0}(x, y)=u(x)+\nabla_{y} u^{1}(x, y)+u^{2}(x, y)$, where the triplet $\left(u, u^{1}, u^{2}\right) \in H_{\text {curl }} \times L^{2}\left(\mathbb{R}^{3} ; H_{\#}^{2}(Q)\right) \times\left[L^{2}\left(\mathbb{R}^{3} ; H_{0}^{1}\left(Q_{0}\right)\right)\right]^{3}=: \mathcal{H}$ is a solution to the system

$$
\begin{aligned}
\operatorname{curl}_{x}\left(A^{\mathrm{hom}} \operatorname{curl}_{x} u(x)\right)+u(x) & +\int_{Q_{0}} u^{2}(x, y) d y \\
& =\int_{Q} f(x, y) d y, \quad x \in \mathbb{R}^{3}, \\
\operatorname{curl}_{y}\left(\epsilon_{0}^{-1}(y) \operatorname{curl}_{y} u^{2}(x, y)\right) & +u(x)+\nabla_{y} u^{1}(x, y)+u^{2}(x, y) \\
& =f(x, y), \quad(x, y) \in \mathbb{R}^{3} \times Q_{0}, \\
\operatorname{div}_{y}\left(\nabla_{y} u^{1}(x, y)+u^{2}(x, y)\right) & =0, \quad(x, y) \in \mathbb{R}^{3} \times Q .
\end{aligned}
$$

Here $\left[L^{2}\left(\mathbb{R}^{3}\right)\right]_{\text {sol }}^{3}$ is the subspace of $\left[L^{2}\left(\mathbb{R}^{3}\right)\right]^{3}$ consisting of divergence-free vector fields, $H_{\text {curl }}:=\left\{u \in\left[L^{2}\left(\mathbb{R}^{3}\right)\right]^{3}:\right.$ curl $\left.u \in\left[L^{2}\left(\mathbb{R}^{3}\right)\right]^{3}\right\}$ and $A^{\text {hom }}$ is a symmetric, positive-definite matrix. In the equation (8), we assume that the function $u^{2}$ is set to zero on $Q_{1}$.

Remark 2.2. The matrix $A^{\text {hom }}$ is described by solutions to certain degenerate "cell problems", which are presented, along with the description of $A^{\text {hom }}$, in Lemma 4.4. Therein, we prove the duality relation

$$
A^{\text {hom }}=\left(\epsilon_{\text {stiff }}^{\text {hom }}\right)^{-1},
$$

where the positive-definite matrix $\epsilon_{\text {stiff }}^{\text {hom }}$ is the homogenized coefficient matrix given by

$$
\epsilon_{\text {stiff }}^{\text {hom }} \xi \cdot \xi:=\inf _{\substack{u \in H_{\#}^{1}(Q), \nabla u=-\xi \text { in } Q_{0}}} \int_{Q_{1}} \epsilon_{1}(\xi+\nabla u) \cdot(\xi+\nabla u), \quad \xi \in \mathbb{R}^{3},
$$

which arises in the homogenization of periodic problems with stiff inclusions; see for example [14, Ch. 3].

Remark 2.3. For any two solutions $\left(u, u^{1}, u^{2}\right),\left(v, v^{1}, v^{2}\right) \in \mathcal{H}$ of (6)-(8), the equalities $u=v$ and $\nabla_{y} u^{1}+u^{2}=\nabla_{y} v^{1}+v^{2}$ hold. Conversely, if $\left(u, u^{1}, u^{2}\right) \in \mathcal{H}$ is a solution to (6)-(8), then all $\left(u, v^{1}, v^{2}\right)$ with $\left(v^{1}, v^{2}\right) \in L^{2}\left(\mathbb{R}^{3} ; H_{\#}^{2}(Q)\right) \times$ $\left[L^{2}\left(\mathbb{R}^{3} ; H_{0}^{1}\left(Q_{0}\right)\right)\right]^{3}$ such that $\nabla_{y} v^{1}+v^{2}=\nabla_{y} u^{1}+u^{2}$ are also solutions to (6)-(8). These claims are proved in Proposition 4.8. 
Henceforth, for a linear set $X$, we denote by $[X]^{3}$ the space of vector-valued functions whose components belong to $X$, i.e. for $u \in[X]^{3}, u=\left\{u_{1}, u_{2}, u_{3}\right\}$, where $u_{1}, u_{2}, u_{3} \in X$. Whenever $X$ is a Banach space, we consider $[X]^{3}$ to be endowed with the product topology (which makes it a Banach space). We define the integral of a vector function (such as e.g. in (6)) to be the vector of the integrals of its components. We use the subscript "\#" to indicate spaces of periodic functions, i.e. the closures of the space of periodic $C^{\infty}$-functions with respect to appropriate norms.

§3. Auxiliary results. In this section we establish several technical results which will be employed when passing to the homogenization limit in the Maxwell system (5). We start with a discussion of properties of functions that belong to the spaces

$$
V:=\left\{v \in\left[H_{\#}^{1}(Q)\right]^{3}: \operatorname{curl} v=0 \text { in } Q_{1}\right\}
$$

and $V^{\perp}$, the orthogonal complement of $V$ in $\left[H_{\#}^{1}(Q)\right]^{3}$ with respect to the following equivalent $H^{1}$-norm:

$$
\|v\|_{H}:=\left(\left|\int_{Q} v\right|^{2}+\int_{Q}|\nabla v|^{2}\right)^{1 / 2},
$$

associated with the inner product

$$
(v, w)_{H}:=\left(\int_{Q} v\right) \cdot\left(\int_{Q} w\right)+\int_{Q} \nabla v \cdot \nabla w .
$$

LEMMA 3.1 (Characterization of the space $V$ ). A function $v \in\left[H_{\#}^{1}(Q)\right]^{3}$ belongs to the space $V$ if and only if $v=a+\nabla b+c$ for some $a \in \mathbb{R}^{3}, b \in H_{\#}^{2}(Q)$ and $c \in\left[H_{0}^{1}\left(Q_{0}\right)\right]^{3}$. Furthermore, the choice of $a=a(v)$ is unique and there exists a constant $C>0$ such that

$$
|a(v)| \leqslant C\|v\|_{\left[H_{\#}^{1}(Q)\right]^{3}} \quad \text { for all } v \in V .
$$

Proof. It is clear that any function of the form $v=a+\nabla b+c$ for some $a \in \mathbb{R}^{3}$, $b \in H_{\#}^{2}(Q)$ and $c \in\left[H_{0}^{1}\left(Q_{0}\right)\right]^{3}$ belongs to $V$. Therefore, its only necessary to prove the reverse inclusion.

For a fixed $v \in V$, by definition curl $v=0$ in $Q_{1}$. It is known (see [10, p. 219]) that since $Q_{1}$ is simply connected, $v=a+\nabla b$ in $Q_{1}$ for some $a \in \mathbb{R}^{3}$ and $\dagger b \in H_{\#}^{2}\left(Q_{1}\right)$. Denoting by $\tilde{b} \in H_{\#}^{2}(Q)$ a fixed extension of $b$ to $Q_{0}$ given by the extension theorem for Sobolev functions (see [1, Ch. IV], [20, §1.1.16] and $[27, \mathrm{p} .181])$ and by trivially extending $a$, we introduce the function $\tilde{v}:=a+\nabla \tilde{b}$. The function $c:=v-\tilde{v}$, by construction, belongs to $\left[H_{0}^{1}\left(Q_{0}\right)\right]^{3}$. The uniqueness of $a$ follows from observing that for given $p \in\left[H_{\#}^{1}\left(Q_{1}\right)\right]^{3}, a \in \mathbb{R}^{3}$ satisfying $\nabla p=a$ in $Q_{1}, a$ is necessarily zero by the connectedness of $Q_{1}$.

$\dagger$ The space $H_{\#}^{2}\left(Q_{1}\right)$ is defined to consist of restrictions to $Q_{1}$ of functions from $H_{\#}^{2}(Q)$. 
To end the proof, we mention that $a(v)$ satisfies (10). As smooth functions are dense in $V$ (see for example the proof of Lemma 4.1, Step 2), we only need to show (10) for $\phi \in\left[C_{\#}^{\infty}\left(Q_{1}\right)\right]^{3}$ such that $\operatorname{curl} \phi=0$. This follows from noting that since $Q_{1}$ is simply connected, one can construct $b$ as follows:

$$
b(y):=\int_{\mathcal{C}} \phi \cdot d \tau-\sum_{i=1}^{3} a_{i} y_{i}, \quad y \in Q_{1},
$$

where $\mathcal{C}$ is a smooth path in $Q_{1}$ connecting the points 0 and $y$ whose unit tangent vector at each point is denoted by $\tau$, and the vector $a=\left(a_{1}, a_{2}, a_{3}\right)$ is given by

$$
a_{i}=\int_{0}^{1} \phi_{i}\left(t e_{i}\right) d t, \quad i=1,2,3,
$$

where $e_{i}, i=1,2,3$, are the Euclidean basis vectors. The bound (10) now follows by a standard embedding theorem (see e.g. [1, Ch. VII]).

LEMMA 3.2 (Characterization of the space $V^{\perp}$ ). A function $w \in\left[H_{\#}^{1}(Q)\right]^{3}$ belongs to $V^{\perp}$ if and only if the following two conditions hold.

(i) The average of $w$ over $Q$ is equal to zero:

$$
\int_{Q} w=0
$$

(ii) There exists a vector function $p \in\left[L^{2}\left(Q_{1}\right)\right]^{3}$ such that the identity

$$
\int_{Q} \nabla w \cdot \nabla \phi=\int_{Q_{1}} p \cdot \operatorname{curl} \phi
$$

holds for all $\phi \in\left[H_{\#}^{1}(Q)\right]^{3}$. (In particular, $\operatorname{div} w=0$ in $Q$ and all components of $w$ are harmonic in $Q_{0}$.)

Proof. Suppose that both (i) and (ii) hold for a function $w \in\left[H_{\#}^{1}(Q)\right]^{3}$. Then, for $\phi \in V$, one has

$$
(w, \phi)_{H}=\left(\int_{Q} w\right) \cdot\left(\int_{Q} \phi\right)+\int_{Q} \nabla w \cdot \nabla \phi=\int_{Q_{1}} p \cdot \operatorname{curl} \phi=0
$$

and therefore $w \in V^{\perp}$.

Conversely, notice that $w \in V^{\perp}$ immediately implies (12) by considering the products $(w, \phi)_{H}$, where $\phi$ are constant elements of $V$. To prove the property (ii), we follow the classical description of linear continuous functionals on Sobolev spaces (see e.g. [1, Theorem 3.8]).

Denote by $\mathcal{H}^{1}$ the space $\left[H_{\#}^{1}(Q)\right]^{3} / \mathbb{R}^{3}$ equipped with the following equivalent $H^{1}$-norm:

$$
\|\cdot\|_{\mathcal{H}^{1}}=\left(\|\operatorname{div} \cdot\|_{L^{2}(Q)}^{2}+\|\operatorname{curl} \cdot\|_{\left[L^{2}(Q)\right]^{3}}^{2}\right)^{1 / 2}
$$


HOMOGENIZATION OF THE SYSTEM OF HIGH-CONTRAST MAXWELL EQUATIONS 481

and consider the (isometric) mapping $M: \mathcal{H}^{1} \rightarrow\left[L^{2}(Q)\right]^{4}$ given by $M \phi=$ $(\operatorname{div} \phi, \operatorname{curl} \phi)$ and the functional $f_{w}$ defined on the image of $M$ by the formula

$$
f_{w}(M \phi)=(w, \phi)_{H}, \quad \phi \in\left[H_{\#}^{1}(Q)\right]^{3} / \mathbb{R}^{3} .
$$

The functional $f_{w}$ is linear and bounded, due to the fact that the norm $\|\cdot\|_{\mathcal{H}^{1}}$ is equivalent to $\|\cdot\|_{H}$ on $\left[H_{\#}^{1}(Q)\right]^{3} / \mathbb{R}^{3}$. By the Hahn-Banach theorem, there exists a norm-preserving extension $\tilde{f}_{w}$ of $f_{w}$ to the whole of $\left[L^{2}(Q)\right]^{4}$. Further, by the Riesz representation theorem, there exist $q \in L^{2}(Q)$ and $p \in\left[L^{2}(Q)\right]^{3}$ such that

$$
\begin{aligned}
(w, \phi)_{H} & =f_{w}(M \phi)=\tilde{f}_{w}(M \phi)=\int_{Q}(q, p) \cdot M \phi \\
& =\int_{Q} q \operatorname{div} \phi+\int_{Q} p \cdot \operatorname{curl} \phi \quad \text { for all } \phi \in\left[H_{\#}^{1}(Q)\right]^{3} / \mathbb{R}^{3} .
\end{aligned}
$$

Note that if $(q, p)$ satisfies (14), then so does $(q+c, p+\nabla \omega+d)$ for any $\omega \in H_{\#}^{1}(Q), c \in \mathbb{R}, d \in \mathbb{R}^{3}$.

Setting $\phi=\nabla \chi$, where $\chi \in H_{\#}^{1}(Q)$ is a solution to the problem $\Delta \chi=g$ for an arbitrary function $g \in L^{2}(Q)$ with zero integral over $Q$, we note that $\nabla \chi \in V$ and consequently obtain

$$
0=(w, \phi)_{H}=\int_{Q} q \Delta \chi=\int_{Q} q g \text { for all } g \in L^{2}(Q) / \mathbb{R}
$$

hence, $q=-c$ for some $c \in \mathbb{R}$. Further, considering $\phi \in\left[C_{0}^{\infty}\left(Q_{0}\right)\right]^{3} \subset V$ yields

$$
0=(w, \phi)_{H}=\int_{Q_{0}} p \cdot \operatorname{curl} \phi \quad \text { for all } \phi \in\left[C_{0}^{\infty}\left(Q_{0}\right)\right]^{3}
$$

and hence curl $p=0$ in $Q_{0}$. This implies, in turn, that $p=-\nabla \omega-d$ in $Q_{0}$ for some $\omega \in H_{\#}^{1}(Q), d \in \mathbb{R}^{3}$.

Since, as noted above, the identity (14) holds with $q$ replaced by $q+c$, which is equal to zero, and $p$ replaced by $p+\nabla \omega+d$, which is equal to zero in $Q_{0}$, the proof of the claim (ii) is complete.

LEMMA 3.3. There exists a constant $C$ such that

$$
\|w\|_{H} \leqslant C\left(\int_{Q_{1}}|\operatorname{curl} w|^{2}\right)^{1 / 2} \text { for all } w \in V^{\perp} .
$$

Proof. The identities (12) and (13) imply that for all $w \in V^{\perp}$ one has

$$
\|w\|_{H}^{2}=\left|\int_{Q} \nabla w \cdot \nabla w\right|=\left|\int_{Q_{1}} p \cdot \operatorname{curl} w\right| \leqslant\|p\|_{\left[L^{2}\left(Q_{1}\right)\right]^{3}}\|\operatorname{curl} w\|_{\left[L^{2}\left(Q_{1}\right)\right]^{3}}
$$


Further, the identity (14) with $q=0,\left.p\right|_{Q_{0}}=0$ implies that

$$
\begin{aligned}
\|p\|_{\left[L^{2}\left(Q_{1}\right)\right]^{3}} & =\|p\|_{\left[L^{2}(Q)\right]^{3}}=\|(q, p)\|_{\left[L^{2}(Q)\right]^{4}}=\left\|f_{w}\right\|_{\mathcal{H}^{1^{\star}}} \\
& =\sup _{\phi}\|\phi\|_{\mathcal{H}}^{-1}\left|(w, \phi)_{H}\right| \leqslant C\|w\|_{H}
\end{aligned}
$$

for some $C>0$. Here $f_{w}$ is the functional that was introduced in the proof of Lemma 3.2. Combining the two estimates above yields the claim of the lemma.

Lemma 3.4. For fixed $F \in\left(\left[H_{\#}^{1}(Q)\right]^{3}\right)^{*}$, the space of bounded linear functionals on $\left[H_{\#}^{1}(Q)\right]^{3}$, the problem

$$
\operatorname{curl}\left(\epsilon_{1}^{-1} \operatorname{curl} w\right)=F
$$

is weakly solvable in $\left[H_{\#}^{1}(Q)\right]^{3}$ if and only if $\langle F, v\rangle=0$ for all $v \in V$. Furthermore, any weak solution to (15) is uniquely described in $V^{\perp}$. Henceforth $\langle\cdot, \cdot\rangle$ denotes the duality relation between $\left(\left[H_{\#}^{1}(Q)\right]^{3}\right)^{*}$ and $\left[H_{\#}^{1}(Q)\right]^{3}$.

Lemma 3.5. Let $u \in\left[L^{2}(Q)\right]^{3}$ satisfy $(u, \Psi)_{\left[L^{2}(Q)\right]^{3}}=0$ for all $\Psi \in$ $\left[L^{2}(Q)\right]^{3}$ such that $\operatorname{curl}\left(\epsilon_{1}^{-1 / 2} \Psi\right)=0$. Then $u=\epsilon_{1}^{-1 / 2}$ curl $w$ for some $w \in$ $\left[H_{\#}^{1}(Q)\right]^{3}$. The choice of $w$ is unique up to an element of $V$.

Lemmas 3.4 and 3.5 are a consequence of Lemma 3.3 and were first proved by Kamotski and Smyshlyaev, see [17], in the more general context of equations with "partial degeneracies". For completeness, we next present the related proofs.

Proof of Lemma 3.4. Let $w$ be a solution of (15) and let $v \in V$. Then, using the definition (9) of the space $V$, we have

$$
\langle F, v\rangle=\int_{Q} \epsilon_{1}^{-1} \operatorname{curl} w \cdot \operatorname{curl} v=0 .
$$

Conversely, let $\langle F, v\rangle=0$ hold for all $v \in V$ and seek a solution $v \in\left[H_{\#}^{1}(Q)\right]^{3}$ to (15). By (16), the identity (15) holds automatically for all $v \in V$; therefore, it is sufficient to verify it for all $v \in V^{\perp}$. Also, by seeking the solution $w$ to belong to $V^{\perp}$ it remains to show that, in the Hilbert space $V^{\perp}$ with the inherited $\left[H_{\#}^{1}(Q)\right]^{3}$-norm $\|\cdot\|_{H}$, the problem (15) satisfies the conditions of the LaxMilgram lemma; see for example [11]. First, the bilinear form

$$
B(w, v):=\int_{Q} \epsilon_{1}^{-1} \operatorname{curl} w \cdot \operatorname{curl} v
$$

is shown to be bounded in $V^{\perp}$, i.e. with some $C>0$ one has

$$
|B(w, v)| \leqslant C\|w\|_{H}\|v\|_{H} \quad \text { for all } v, w \in V^{\perp} .
$$


This follows from the assumption that $\epsilon_{1}^{-1} \in L^{\infty}(Q)$. Further, the form $B$ is coercive, i.e. for some $v>0$ the bound

$$
B[v, v] \geqslant v\|v\|_{H}^{2} \quad \text { for all } v \in V^{\perp}
$$

holds. Indeed, we have

$$
B(v, v):=\int_{Q} \epsilon_{1}^{-1} \operatorname{curl} v \cdot \operatorname{curl} v=\left\|\epsilon_{1}^{-1 / 2} \operatorname{curl} v\right\|_{\left[L^{2}\left(Q_{1}\right)\right]^{3}}^{2} \geqslant v\|v\|_{H}^{2} .
$$

In the last inequality we have used the fact that $\epsilon_{1}^{-1 / 2}$ is positive on $Q_{1}$ and Lemma 3.3. Finally, by the Lax-Milgram lemma, there exists a unique solution to the problem

$$
w \in V^{\perp}: \quad B(w, v)=\langle F, v\rangle \quad \text { for all } v \in V^{\perp}
$$

and hence to (15).

Proof of Lemma 3.5. Let $u$ satisfy the assumption of the lemma. Defining the right-hand side $F \in\left(\left[H_{\#}^{1}(Q)\right]^{3}\right)^{*}$ by $F:=\operatorname{curl}\left(\epsilon_{1}^{-1 / 2} u\right)$, we see that $F$ satisfies the condition $\langle F, v\rangle=0$ for all $v \in V$, since for such $v$ one has

$$
\langle F, v\rangle=\left\langle\operatorname{curl}\left(\epsilon_{1}^{-1 / 2} u\right), v\right\rangle=\int_{Q} \epsilon_{1}^{-1 / 2} u \cdot \operatorname{curl} v=0 .
$$

By Lemma 3.4, there exists a unique $w \in V^{\perp}$ such that

$$
\operatorname{curl}\left(\epsilon_{1}^{-1} \operatorname{curl} w\right)=\operatorname{curl}\left(\epsilon_{1}^{-1 / 2} u\right) .
$$

It remains to show that $u=\epsilon_{1}^{-1 / 2}$ curl $w$. Indeed, we have

$$
\begin{gathered}
\left\|u-\epsilon_{1}^{-1 / 2} \operatorname{curl} w\right\|_{\left[L^{2}(Q)\right]^{3}}^{2}=\left(u, u-\epsilon_{1}^{-1 / 2} \operatorname{curl} w\right)_{\left[L^{2}(Q)\right]^{3}} \\
-\left(\epsilon_{1}^{-1 / 2} \operatorname{curl} w, u-\epsilon_{1}^{-1 / 2} \operatorname{curl} w\right)_{\left[L^{2}(Q)\right]^{3}}=: S_{1}+S_{2} .
\end{gathered}
$$

It follows from (17) that $S_{1}=0$, since for $\Psi:=u-\epsilon_{1}^{-1 / 2}$ curl $w$ one has $\operatorname{curl}\left(\epsilon_{1}^{-1 / 2} \Psi\right)=0$ and therefore $(u, \Psi)_{\left[L^{2}(Q)\right]^{3}}=0$ by the assumption on $u$. On the other hand,

$$
S_{2}=\int_{Q} \epsilon^{-1 / 2} \operatorname{curl} w \cdot \Psi=\int_{Q} \operatorname{curl} w \cdot \epsilon_{1}^{-1 / 2} \Psi=:\left\langle\operatorname{curl}\left(\epsilon_{1}^{-1 / 2} \Psi\right), w\right\rangle=0
$$

by (17). Hence, $\left\|u-\epsilon_{1}^{-1 / 2} \operatorname{curl} w\right\|_{\left[L^{2}(Q)\right]^{3}}=0$, yielding the desired result.

The above construction also ensures that $w$ is determined uniquely up to any function from $V$; in particular, it is unique in $V^{\perp}$. 
§. Homogenization. This section contains the proof of the main result (Theorem 2.1). We have structured our presentation in two parts: the analysis of compactness properties of the sequence of solutions $u^{\eta}$ and the passage to the limit as $\eta \rightarrow 0$ in the weak formulation of (5).

\subsection{Two-scale compactness of solution sequences.}

LEMMA 4.1. Suppose that a sequence $f^{\eta} \in\left[L^{2}\left(\mathbb{R}^{3}\right)\right]_{\text {sol }}^{3}$ weakly two-scale converges to $f \in\left[L^{2}\left(\mathbb{R}^{3} \times Q\right)\right]^{3}$. Then the sequence $u^{\eta} \in\left[H^{1}\left(\mathbb{R}^{3}\right)\right]^{3}$ of solutions to (5) weakly two-scale converges, up to a subsequence that we do not relabel, to $u^{0}(x, y) \in L^{2}\left(\mathbb{R}^{3} ; V\right)$, where

$$
\operatorname{div}_{y} u^{0}=0
$$

and

$$
u^{0}(x, y)=u(x)+\nabla_{y} u^{1}(x, y)+u^{2}(x, y)
$$

for some $\left(u, u^{1}, u^{2}\right) \in H_{\text {curl }} \times L^{2}\left(\mathbb{R}^{3} ; H_{\#}^{2}(Q)\right) \times\left[L^{2}\left(\mathbb{R}^{3} ; H_{0}^{1}\left(Q_{0}\right)\right)\right]^{3}=: \mathcal{H}$. Furthermore, one has

$$
\begin{gathered}
\eta \operatorname{curl} u^{\eta}(x) \stackrel{2}{\rightarrow} \operatorname{curl}_{y} u^{0}(x, y), \\
\epsilon_{1}^{-1}(x / \eta) \operatorname{curl} u^{\eta}(x) \stackrel{2}{\rightarrow} \epsilon_{1}^{-1}(y)\left[\operatorname{curl} u(x)+\operatorname{curl}_{y} w(x, y)\right]
\end{gathered}
$$

as $\eta \rightarrow 0$. Henceforth we denote by $\stackrel{2}{\rightarrow}$ and $\stackrel{2}{\rightarrow}$ the weak and strong two-scale convergence, respectively. The function $w \in L^{2}\left(\mathbb{R} ; V^{\perp}\right)$ in (21) is the unique solution in $V^{\perp}$ to the problem

$$
\operatorname{curl}_{y}\left(\epsilon_{1}^{-1}(y)\left[\operatorname{curl}_{y} w(x, y)+\operatorname{curl} u(x)\right]\right)=0 .
$$

In particular, one has

$$
w(x, y)=M(y) \operatorname{curl} u(x) \text { for a.e. }(x, y) \in \mathbb{R}^{3} \times Q,
$$

where $M \in\left[H_{\#}^{1}(Q)\right]^{3 \times 3}$ is a matrix-valued function whose columns $M^{r}, r=1$, 2,3 , are the unique solutions in $V^{\perp}$ to the problem

$$
\operatorname{curl}\left(\epsilon_{1}^{-1}\left[\operatorname{curl} M^{r}+e_{r}\right]\right)=0 .
$$

Here, as before, $e_{r}, r=1,2,3$, are the Euclidean basis vectors.

Remark 4.2. Note that equations (18) and (19) give (8).

Proof. Let $u^{\eta}$ be the sequence of solutions given in the hypothesis of the lemma. We begin by noting that there exists a constant $C>0$ such that

$$
\begin{aligned}
\left\|u^{\eta}\right\|_{\left[L^{2}\left(\mathbb{R}^{3}\right)\right]^{3}} & \leqslant C\left\|f^{\eta}\right\|_{\left[L^{2}\left(\mathbb{R}^{3}\right)\right]^{3}}, \\
\left\|\eta \operatorname{curl} u^{\eta}\right\|_{\left[L^{2}\left(\mathbb{R}^{3}\right)\right]^{3}} & \leqslant C\left\|f^{\eta}\right\|_{\left[L^{2}\left(\mathbb{R}^{3}\right)\right]^{3}}, \\
\left\|\epsilon_{1}^{-1 / 2} \operatorname{curl} u^{\eta}\right\|_{\left[L^{2}\left(\mathbb{R}^{3}\right)\right]^{3}} & \leqslant C\left\|f^{\eta}\right\|_{\left[L^{2}\left(\mathbb{R}^{3}\right)\right]^{3}} .
\end{aligned}
$$


Indeed, as $u^{\eta}$ is a weak solution to (5), by definition $u^{\eta}$ satisfies

$$
\begin{gathered}
\int_{\mathbb{R}^{3}}\left(\epsilon_{1}^{-1}+\eta^{2} \epsilon_{0}^{-1}\right)(x / \eta) \operatorname{curl} u^{\eta}(x) \cdot \operatorname{curl} \phi(x) d x+\int_{\mathbb{R}^{3}} u^{\eta}(x) \cdot \phi(x) d x \\
=\int_{\mathbb{R}^{3}} f^{\eta}(x) \cdot \phi(x) d x \quad \text { for all } \phi \in\left[H^{1}\left(\mathbb{R}^{3}\right)\right]^{3} .
\end{gathered}
$$

Setting $\phi=u^{\eta}$ in (25) yields the above inequalities.

As bounded sequences in $\left[L^{2}\left(\mathbb{R}^{3}\right)\right]^{3}$ have two-scale convergent subsequences we find, upon extraction of a suitable subsequence, that

$$
u^{\eta} \stackrel{2}{\rightarrow} u^{0}, \quad \eta \operatorname{curl} u^{\eta} \stackrel{2}{\rightarrow} \Xi^{0}, \quad \epsilon_{1}^{-1 / 2}(\cdot / \eta) \operatorname{curl} u^{\eta} \stackrel{2}{\rightarrow} \xi^{0}
$$

for some $u^{0}, \Xi^{0}, \xi^{0} \in\left[L^{2}\left(\mathbb{R}^{3} \times Q\right)\right]^{3}$. What remains is to show that these limit functions satisfy the announced properties of the lemma. The proofs of these facts shall be presented in several distinct steps.

Step 1. Here we shall show that $u^{0}$ belongs to $L^{2}\left(\mathbb{R}^{3} ; V\right)$ and that equations (18)-(20) hold. Suppose that $\Psi \in\left[C_{0}^{\infty}\left(\mathbb{R}^{3} ; C_{\#}^{\infty}(Q)\right)\right]^{3}$; then, passing to the limit as $\eta \rightarrow 0$ in the identity

$$
\begin{aligned}
\int_{\mathbb{R}^{3}} & \eta \operatorname{curl} u^{\eta}(x) \cdot \Psi\left(x, \frac{x}{\eta}\right) d x \\
& =\int_{\mathbb{R}^{3}} u^{\eta}(x) \cdot\left[\eta \operatorname{curl}_{x} \Psi\left(x, \frac{x}{\eta}\right)+\operatorname{curl}_{y} \Psi\left(x, \frac{x}{\eta}\right)\right] d x
\end{aligned}
$$

yields

$$
\int_{\mathbb{R}^{3}} \int_{Q} \Xi^{0}(x, y) \cdot \Psi(x, y) d y d x=\int_{\mathbb{R}^{3}} \int_{Q} u^{0}(x, y) \cdot \operatorname{curl}_{y} \Psi(x, y) d y d x,
$$

which implies equation (20). By a similar passage to the limit in the identity

$$
\begin{aligned}
0 & =\int_{\mathbb{R}^{3}} \eta u^{\eta}(x) \cdot \nabla \Psi\left(x, \frac{x}{\eta}\right) d x \\
& =\int_{\mathbb{R}^{3}} u^{\eta}(x) \cdot\left[\eta \nabla_{x} \Psi\left(x, \frac{x}{\eta}\right)+\nabla_{y} \Psi\left(x, \frac{x}{\eta}\right)\right] d x,
\end{aligned}
$$

which holds as $\operatorname{div} u^{\eta}=0$, one finds that

$$
0=\int_{\mathbb{R}^{3}} \int_{Q} u^{0}(x, y) \cdot \nabla_{y} \Psi(x, y)
$$

which implies (18). The above two observations concerning $\operatorname{curl}_{y} u^{0}$ and $\operatorname{div}_{y} u^{0}$ imply, in particular, that $u^{0}(x, \cdot) \in\left[H_{\#}^{1}(Q)\right]^{3}$ for almost every $x \in \mathbb{R}^{3}$. What remains of this step is to show that $u^{0} \in L^{2}\left(\mathbb{R}^{3} ; V\right)$, which, in turn, by Lemma 3.1, implies equation (19) and the fact that $u$ (given by (11)) belongs 
to $\left[L^{2}\left(\mathbb{R}^{3}\right)\right]^{3}$. To accomplish this we note that, on the one hand, the convergence

$$
\lim _{\eta \rightarrow 0} \int_{\mathbb{R}^{3}} \eta \epsilon_{1}^{-1 / 2}\left(\frac{x}{\eta}\right) \operatorname{curl} u^{\eta}(x) \cdot \Psi\left(x, \frac{x}{\eta}\right) d x=0
$$

holds, due to the $L^{2}$-boundedness of the sequence $\epsilon_{1}^{-1 / 2}(\cdot / \eta) \operatorname{curl} u^{\eta}$. On the other hand, by (20) one has

$$
\begin{aligned}
\lim _{\eta \rightarrow 0} & \int_{\mathbb{R}^{3}} \eta \epsilon_{1}^{-1 / 2}\left(\frac{x}{\eta}\right) \operatorname{curl} u^{\eta}(x) \cdot \Psi\left(x, \frac{x}{\eta}\right) d x \\
& =\int_{\mathbb{R}^{3}} \int_{Q} \epsilon_{1}^{-1 / 2}(y) \operatorname{curl}_{y} u^{0}(x, y) \cdot \Psi(x, y) d y d x .
\end{aligned}
$$

Combining (26) and (27) and using the arbitrary choice of the test function $\Psi$ yields

$$
\epsilon_{1}^{-1 / 2}(y) \operatorname{curl}_{y} u^{0}(x, y) \text { for a.e. } x, y \in \mathbb{R}^{3} \times Q
$$

and hence $u^{0}(x, \cdot) \in V$; see (9).

Step 2. To prove (22)-(24), which is the subject of Step 3 below, we shall require the following identity:

$$
\begin{aligned}
\int_{\mathbb{R}^{3}} \int_{Q} \xi^{0}(x, y) \cdot \Psi(x, y) d y d x \\
\quad=\int_{\mathbb{R}^{3}} \int_{Q} \epsilon_{1}^{-1 / 2}(y) u^{0}(x, y) \cdot \operatorname{curl}_{x} \Psi(x, y) d y d x
\end{aligned}
$$

for all test functions $\Psi \in W \cap\left[C_{0}^{\infty}\left(\mathbb{R}^{3} ; L^{2}(Q)\right)\right]^{3}$, where

$$
W:=\left\{\Psi \in\left[L^{2}\left(\mathbb{R}^{3} \times Q\right)\right]^{3}: \operatorname{curl}_{y}\left(\epsilon_{1}^{-1 / 2}(y) \Psi(x, y)\right)=0 \text { for a.e. } x \in \mathbb{R}^{3}\right\} .
$$

We shall now prove (28). Let $\varphi_{k}=\varphi_{k}(x, y), k \in \mathbb{N}$, be the sequence of functions obtained by the convolution ("mollification") of $\epsilon_{1}^{-1 / 2}(\cdot) \Psi(x, \cdot)$ with functions $\omega(k \cdot)$ such that $\omega$ is a $C_{0}^{\infty}$-function with integral equal to unity:

$$
\varphi_{k}(x, y)=\int_{\mathbb{R}^{3}} \epsilon_{1}^{-1 / 2}(y-\xi) \Psi(x, y-\xi) \omega(k \xi) d \xi, \quad k \in \mathbb{N}
$$

where $\epsilon_{1}^{-1 / 2}(\cdot) \Psi(x, \cdot)$ is extended to $\mathbb{R}^{3}$ by $Q$-periodicity. Let us recall some properties of these mollifications that we shall employ. Namely, we notice that $\varphi_{k} \in\left[C_{0}^{\infty}\left(\mathbb{R}^{3} ; C_{\#}^{\infty}(Q)\right)\right]^{3}$ and that the sequence $\varphi_{k}(x, \cdot)$ converges to the function $\varphi:=\epsilon^{-1 / 2} \Psi$ strongly in $\left[C\left(K ; L^{2}(Q)\right)\right]^{3}$, where the compact set $K$ is the support of $\Psi$ with respect to $x$. Similarly, the sequence $\operatorname{curl}_{x} \varphi_{k}(x, \cdot)$ converges to $\operatorname{curl}_{x} \varphi=\epsilon^{-1 / 2} \operatorname{curl}_{x} \Psi$ strongly in $\left[C\left(K ; L^{2}(Q)\right)\right]^{3}$. Moreover, one has $\operatorname{curl}_{y} \varphi_{k}(x, y)=0$ for all $k$ and, by integration by parts, we have the identity

$$
\int_{\mathbb{R}^{3}} \operatorname{curl} u^{\eta}(x) \cdot \varphi_{k}\left(x, \frac{x}{\eta}\right) d x=\int_{\mathbb{R}^{3}} u^{\eta}(x) \cdot \operatorname{curl}_{x} \varphi_{k}\left(x, \frac{x}{\eta}\right) d x .
$$


We wish to pass to the limit $k \rightarrow \infty$ in (29), which requires proving that for a fixed $\eta$, the functions $\varphi_{k}(x, x / \eta)\left(\right.$ respectively $\left.\operatorname{curl}_{x} \varphi_{k}(x, x / \eta)\right)$ converge strongly in $\left[L^{2}\left(\mathbb{R}^{3}\right)\right]^{3}$ to $\varphi(x, x / \eta)$ (respectively $\left.\operatorname{curl}_{x} \varphi(x, x / \eta)\right)$. We will only show this for $\varphi_{k}(x, x / \eta)$ as the argument for $\operatorname{curl}_{x} \varphi_{k}(x, x / \eta)$ is identical. Recalling that $K$ denotes the support of $\epsilon^{-1 / 2}(x) \Psi(x, y)$ with respect to $x$ and taking $\left\{Q_{i}\right\}_{i=1}^{n}$ to be a finite set of translations of $Q$ which covers $\eta^{-1} K$, we find that

$$
\begin{aligned}
\int_{\mathbb{R}^{3}}\left|\varphi_{k}\left(x, \frac{x}{\eta}\right)-\varphi\left(x, \frac{x}{\eta}\right)\right|^{2} d x=\int_{K}\left|\varphi_{k}\left(x, \frac{x}{\eta}\right)-\varphi\left(x, \frac{x}{\eta}\right)\right|^{2} d x \\
=\int_{\eta^{-1} K} \eta^{3}\left|\varphi_{k}(\eta y, y)-\varphi(\eta y, y)\right|^{2} d y \\
\leqslant \eta^{3} \int_{\eta^{-1}} \sup _{x \in K}\left|\varphi_{k}(x, y)-\varphi(x, y)\right|^{2} d y \\
=\eta^{3} \sum_{i=1}^{n} \sup _{x \in K} \int_{Q_{i}}\left|\varphi_{k}(x, y)-\varphi(x, y)\right|^{2} d y \rightarrow 0
\end{aligned}
$$

as $k \rightarrow \infty$ by the properties of the mollification. Therefore, we can replace (29) with

$$
\int_{\mathbb{R}^{3}} \epsilon_{1}^{-1}\left(\frac{x}{\eta}\right) \operatorname{curl} u^{\eta}(x) \cdot \Psi\left(x, \frac{x}{\eta}\right) d x=\int_{\mathbb{R}^{3}} u^{\eta}(x) \cdot \epsilon_{1}^{-1}\left(\frac{x}{\eta}\right) \operatorname{curl}_{x} \Psi\left(x, \frac{x}{\eta}\right) d x
$$

and, by passing to the limit in (30) as $\eta \rightarrow 0$, we obtain (28).

Step 3. Here we shall prove (22)-(24). Let us first show that

$$
\begin{gathered}
\int_{\mathbb{R}^{3}} \int_{Q} \epsilon_{1}^{-1 / 2}(y) \nabla_{y} u^{1}(x, y) \cdot \operatorname{curl}_{x} \Psi(x, y) d y d x \\
=0 \quad \text { for all } \Psi \in W \cap\left[C_{0}^{\infty}\left(\mathbb{R}^{3} ; L^{2}(Q)\right)\right]^{3} .
\end{gathered}
$$

Indeed, by integration by parts, along with the identity $\operatorname{div}_{y} \operatorname{curl}_{x}=-\operatorname{div}_{x} \operatorname{curl}_{y}$, we find that

$$
\begin{aligned}
\int_{\mathbb{R}^{3}} & \int_{Q} \epsilon_{1}^{-1 / 2}(y) \nabla_{y} u^{1}(x, y) \cdot \operatorname{curl}_{x} \Psi(x, y) d y d x \\
& =\int_{\mathbb{R}^{3}} \int_{Q} u^{1}(x, y) \operatorname{div}_{y}\left(\epsilon_{1}^{-1 / 2}(y) \operatorname{curl}_{x} \Psi(x, y)\right) d y d x \\
& =\int_{\mathbb{R}^{3}} \int_{Q} u^{1}(x, y) \operatorname{div}_{y} \operatorname{curl}_{x}\left(\epsilon_{1}^{-1 / 2}(y) \Psi(x, y)\right) d y d x \\
& =-\int_{\mathbb{R}^{3}} \int_{Q} u^{1}(x, y) \operatorname{div}_{x} \operatorname{curl}_{y}\left(\epsilon_{1}^{-1 / 2}(y) \Psi(x, y)\right) d y d x=0
\end{aligned}
$$


for $\Psi \in W \cap\left[C_{0}^{\infty}\left(\mathbb{R}^{3} ; L^{2}(Q)\right)\right]^{3}$. Substituting the representation (19) into (28) and noting (31), we find that

$$
\begin{gathered}
\int_{\mathbb{R}^{3}} \int_{Q} \xi^{0}(x, y) \cdot \Psi(x, y) d y d x=\int_{\mathbb{R}^{3}} \int_{Q} \epsilon_{1}^{-1 / 2}(y) u(x) \cdot \operatorname{curl}_{x} \Psi(x, y) d y d x \\
\text { for all } \Psi \in W \cap\left[C_{0}^{\infty}\left(\mathbb{R}^{3} ; L^{2}(Q)\right)\right]^{3} .
\end{gathered}
$$

In fact, as all smooth vector functions in $\mathbb{R}^{3}$ can be approximated in $\left[H^{1}\left(\mathbb{R}^{3}\right)\right]^{3}$ by smooth functions with compact support, we find that

$$
\begin{array}{r}
\int_{\mathbb{R}^{3}} \int_{Q} \xi^{0}(x, y) \cdot \Psi(x, y) d y d x=\int_{\mathbb{R}^{3}} \int_{Q} \epsilon_{1}^{-1 / 2}(y) u(x) \cdot \operatorname{curl}_{x} \Psi(x, y) d y d x \\
\quad \text { for all } \Psi \in W \cap\left[C^{\infty}\left(\mathbb{R}^{3} ; L^{2}(Q)\right)\right]^{3} . \text { (32) }
\end{array}
$$

Assuming for the moment that curl $u \in\left[L^{2}\left(\mathbb{R}^{3}\right)\right]^{3}$, an integration by parts in (32) and a standard density argument imply that

$$
\begin{gathered}
\int_{Q}\left[\xi^{0}(x, y)-\epsilon_{1}^{-1 / 2} \operatorname{curl}(x)\right] \cdot \Psi(x, y) d y=0 \\
\text { for all } \Psi \in W \text { for a.e. } x \in \mathbb{R}^{3} .
\end{gathered}
$$

In view of Lemma 3.5, we now arrive at

$\xi^{0}(x, y)=\epsilon_{1}^{-1 / 2}(y) \operatorname{curl} u(x)+\epsilon_{1}^{-1 / 2}(y) \operatorname{curl}_{y} w(x, y) \quad$ for a.e. $(x, y) \in \mathbb{R}^{3} \times Q$

for some $w \in L^{2}\left(\mathbb{R}^{3} ; V^{\perp}\right)$, which gives (21). Finally we note, by passing to the limit as $\eta \rightarrow 0$ in (25) with test functions of the form $\phi=\eta \Psi(\cdot, \cdot / \eta)$ for arbitrary $\Psi \in\left[C_{0}^{\infty}\left(\mathbb{R}^{3} ; C_{\#}^{\infty}(Q)\right)\right]^{3}$, that

$$
\operatorname{curl}_{y}\left(\epsilon_{1}^{-1 / 2}(y) \xi^{0}(x, y)\right)=0 \text { for a.e. }(x, y) \in \mathbb{R}^{3} \times Q,
$$

which, along with (33), gives (22). The decomposition (23) then simply follows, since the existence of solutions to (24) is guaranteed by Lemma 3.4.

Step 4. Here we show that the assumption we used in Step 2, namely that $u \in H_{\text {curl }}$, holds. To this end, we prove the following proposition.

Proposition 4.3. For all $\phi \in\left[C_{0}^{\infty}\left(\mathbb{R}^{3}\right)\right]^{3}$ there exists $\Psi_{\phi} \in W \cap$ $\left[C^{\infty}\left(\mathbb{R}^{3} ; L^{2}(Q)\right)\right]^{3}$ such that

$$
\operatorname{curl} \phi(x)=\int_{Q} \operatorname{curl}_{x}\left(\epsilon_{1}^{-1 / 2}(y) \Psi_{\phi}(x, y)\right) d y, \quad x \in \mathbb{R}^{3} .
$$

Further, for all functions $w \in W$ there exists $C(w)>0$ such that

$$
\int_{\mathbb{R}^{3}} \int_{Q} w(x, y) \Psi_{\phi}(x, y) d y d x \leqslant C(w)\|\phi\|_{\left[L^{2}\left(\mathbb{R}^{3}\right)\right]^{3}} \text { for all } \phi \in\left[C_{0}^{\infty}\left(\mathbb{R}^{3}\right)\right]^{3} .
$$


Notice that if Proposition 4.3 holds, for an arbitrary $\phi \in\left[C_{0}^{\infty}\left(\mathbb{R}^{3}\right)\right]^{3}$ we choose the corresponding $\Psi_{\phi}$ as the test function in (32) which will subsequently yield

$$
\int_{\mathbb{R}^{3}} u(x) \cdot \operatorname{curl} \phi(x) d x=l(\phi),
$$

where the linear functional $l:\left[C_{0}^{\infty}\left(\mathbb{R}^{3}\right)\right]^{3} \rightarrow \mathbb{R}^{3}$ is given by

$$
l(\phi):=\int_{\mathbb{R}^{3}} \int_{Q} \xi^{0}(x, y) \Psi_{\phi}(x, y) d y d x .
$$

The inequality (36), which holds since $\xi^{0} \in W$ (see (34)), implies that $l$ is bounded and can therefore be extended to a bounded functional on $\left[L^{2}\left(\mathbb{R}^{3}\right)\right]^{3}$. Hence, by the Riesz representation theorem, we find that curl $u \in\left[L^{2}\left(\mathbb{R}^{3}\right)\right]^{2}$.

Proof of Proposition 4.3. For fixed $\phi \in\left[C_{0}^{\infty}\left(\mathbb{R}^{3}\right)\right]^{3}$, let $\phi^{0} \in\left[H^{1}\left(\mathbb{R}^{3}\right)\right]^{3} \cap$ $\left[C^{\infty}\left(\mathbb{R}^{3}\right)\right]^{3}$ be a solution to the problem

$$
\operatorname{curl}\left(A^{\text {hom }} \operatorname{curl} \phi^{0}\right)=\operatorname{curl} \phi, \quad \operatorname{div} \phi^{0}=0,
$$

where the positive-definite matrix $A^{\text {hom }}$ is given by Lemma 4.4. By a standard Fourier transform argument, the solution $\phi^{0}$ exists and is unique in the class indicated. Next, take $\phi^{1} \in L^{2}\left(\mathbb{R}^{3} ; V^{\perp}\right) \cap\left[C^{\infty}\left(\mathbb{R}^{3} ; H_{\#}^{1}(Q)\right)\right]^{3}$ to be the solution to

$$
\operatorname{curl}_{y}\left(\epsilon_{1}^{-1}(y)\left[\operatorname{curl}_{y} \phi^{1}(x, y)+\operatorname{curl} \phi^{0}(x)\right]\right)=0,
$$

whose existence is guaranteed by Lemma 3.4. Setting

$$
\Psi_{\phi}(x, y):=\epsilon_{1}^{-1 / 2}(y)\left[\operatorname{curl}_{y} \phi^{1}(x, y)+\operatorname{curl} \phi^{0}(x)\right], \quad(x, y) \in \mathbb{R}^{3} \times Q,
$$

we see by construction that $\Psi \in W \cap\left[C^{\infty}\left(\mathbb{R}^{3} ; L^{2}(Q)\right)\right]^{3}$ and

$$
\int_{Q} \epsilon_{1}^{-1 / 2}(y) \Psi(x, y) d y=A^{\text {hom }} \operatorname{curl} \phi^{0}, \quad x \in \mathbb{R}^{3},
$$

where the last equality is derived from Lemma 4.4 below and by observing that $\phi^{1}(x, y)=M(y) \operatorname{curl} \phi^{0}$ for a matrix $M$ whose columns $M^{r}$ solve the cell problem (24). Finally, equations (37) and (39) imply (35).

The inequality (36) results from the following observations. For each $w \in W$ we obtain, in view of the representation (38), that

$$
\begin{aligned}
& \left|\int_{\mathbb{R}^{3}} \int_{Q} w(x, y) \cdot \Psi_{\phi}(x, y) d y d x\right| \\
& \quad=\left|\int_{\mathbb{R}^{3}} \int_{Q} w(x, y) \cdot \epsilon_{1}^{-1 / 2}(y) \operatorname{curl} \phi^{0}(x) d y d x\right| \\
& \quad \leqslant\left(\int_{\mathbb{R}^{3}}\left|\operatorname{curl} \phi^{0}(x)\right|^{2} d x\right)^{1 / 2}\left(\int_{\mathbb{R}^{3}} \int_{Q}\left|\epsilon_{1}^{-1 / 2}(y) w(x, y)\right|^{2} d y d x\right)^{1 / 2} .
\end{aligned}
$$


Furthermore, by (37) and the fact that $A^{\text {hom }}$ is positive definite, there exists a constant $C$ such that

$$
\begin{aligned}
\int_{\mathbb{R}^{3}}\left|\operatorname{curl} \phi^{0}\right|^{2} & \leqslant C \int_{\mathbb{R}^{3}} A^{\text {hom }} \operatorname{curl} \phi^{0} \cdot \operatorname{curl} \phi^{0}=C \int_{\mathbb{R}^{3}} \phi \cdot \operatorname{curl} \phi^{0} \\
& \leqslant C\left(\int_{\mathbb{R}^{3}}\left|\operatorname{curl} \phi^{0}\right|^{2}\right)^{1 / 2}\left(\int_{\mathbb{R}^{3}}|\phi|^{2}\right)^{1 / 2} .
\end{aligned}
$$

This completes the proof of Lemma 4.1.

4.2. Proof of Theorem 2.1. We first consider the case $f^{\eta} \stackrel{2}{\rightarrow} f^{0}$ and let $u^{\eta}$, $u^{0}$ be given by Lemma 4.1. Then, for $\phi^{0} \in C_{0}^{\infty}\left(\mathbb{R}^{3} ; V \cap\left[C_{\#}^{\infty}(Q)\right]^{3}\right)$, we set $\phi=\phi^{0}(\cdot, \cdot / \eta)$ as the test function in (25). We note, by the convergence (21), that

$$
\begin{aligned}
\int_{\mathbb{R}^{3}} & \epsilon_{1}^{-1}\left(\frac{x}{\eta}\right) \operatorname{curl} u^{\eta}(x) \cdot \operatorname{curl} \phi(x) d x \\
= & \int_{\mathbb{R}^{3}} \epsilon_{1}^{-1}\left(\frac{x}{\eta}\right) \operatorname{curl} u^{\eta}(x) \cdot\left[\operatorname{curl}_{x} \phi^{0}\left(x, \frac{x}{\eta}\right)+\eta^{-1} \operatorname{curl}_{y} \phi^{0}\left(x, \frac{x}{\eta}\right)\right] d x \\
& =\int_{\mathbb{R}^{3}} \epsilon_{1}^{-1}\left(\frac{x}{\eta}\right) \operatorname{curl} u^{\eta}(x) \cdot \operatorname{curl}_{x} \phi^{0}\left(x, \frac{x}{\eta}\right) d x \\
& \stackrel{\eta \rightarrow 0}{\longrightarrow} \int_{\mathbb{R}^{3}} \int_{Q} \epsilon_{1}^{-1}(y)\left(\operatorname{curl} u(x)+\operatorname{curl}_{y} u^{1}(x, y)\right) \cdot \operatorname{curl}_{x} \phi^{0}(x, y) d y d x .
\end{aligned}
$$

We similarly note, by (20), that

$$
\begin{aligned}
\int_{\mathbb{R}^{3}} & \eta^{2} \epsilon_{0}^{-1}\left(\frac{x}{\eta}\right) \operatorname{curl} u^{\eta}(x) \cdot \operatorname{curl} \phi(x) d x \\
& =\int_{\mathbb{R}^{3}} \eta \epsilon_{0}^{-1}\left(\frac{x}{\eta}\right) \operatorname{curl} u^{\eta}(x) \cdot \operatorname{curl}_{y} \phi^{0}\left(x, \frac{x}{\eta}\right) d x+o(\eta) \\
& \stackrel{\eta \rightarrow 0}{\longrightarrow} \int_{\mathbb{R}^{3}} \int_{Q} \epsilon_{0}^{-1}(y) \operatorname{curl}_{y} u^{0}(x, y) \cdot \operatorname{curl}_{y} \phi^{0}(x, y) d y d x .
\end{aligned}
$$

Therefore, passing to the limit in (25) for such functions $\phi$ yields

$$
\begin{aligned}
\int_{\mathbb{R}^{3}} & \int_{Q} \epsilon_{1}^{-1}(y)\left(\operatorname{curl} u(x)+\operatorname{curl}_{y} u^{1}(x, y)\right) \cdot \operatorname{curl}_{x} \phi^{0}(x, y) d y d x \\
& +\int_{\mathbb{R}^{3}} \int_{Q} \epsilon_{0}^{-1}(y) \operatorname{curl}_{y} u^{0}(x, y) \cdot \operatorname{curl}_{y} \phi^{0}(x, y) d y d x \\
& +\int_{\mathbb{R}^{3}} \int_{Q} u^{0}(x, y) \cdot \phi^{0}(x, y) d y d x \\
= & \int_{\mathbb{R}^{3}} \int_{Q} f(x, y) \cdot \phi^{0}(x, y) d y d x \text { for all } \phi^{0} \in C_{0}^{\infty}\left(\mathbb{R}^{3} ; V \cap\left[C_{\#}^{\infty}(Q)\right]^{3}\right) .
\end{aligned}
$$

Now we shall use the identity (40) to obtain the equations (6)-(7) of the main theorem. (Recall that the equation (8) was obtained earlier; see Remark 4.2.) 
First, using the equation (23), we find that

$$
\begin{aligned}
\int_{\mathbb{R}^{3}} & \int_{Q} \epsilon_{1}^{-1}(y)\left(\operatorname{curl} u(x)+\operatorname{curl}_{y} u^{1}(x, y)\right) \cdot \operatorname{curl}_{x} \phi^{0}(x, y) d y d x \\
= & \int_{\mathbb{R}^{3}} \int_{Q} \epsilon_{1}^{-1}(y)[I+\operatorname{curl} M(y)] \operatorname{curl} u(x) \cdot \operatorname{curl}_{x} \phi^{0}(x, y) d y d x \\
= & \int_{\mathbb{R}^{3}} A^{\text {hom }} \operatorname{curl} u(x) \cdot \operatorname{curl} \phi(x) d x \\
& +\int_{\mathbb{R}^{3}} \int_{Q} \epsilon_{1}^{-1}(y)[I+\operatorname{curl} M(y)] \operatorname{curl} u(x) \cdot \operatorname{curl}_{x} \nabla_{y} \phi^{1}(x, y) d y d x,
\end{aligned}
$$

where $A^{\text {hom }}$ is given by Lemma 4.4. Let us now show that the second term on the right-hand side of (41) is zero. For functions $\phi^{1} \in C_{0}^{\infty}\left(\mathbb{R}^{3} ; H_{\#}^{2}(Q)\right)$, we find by the identity $\operatorname{curl}_{x} \nabla_{y}=-\operatorname{curl}_{y} \nabla_{x}$ and the equation (24) that

$$
\begin{aligned}
\int_{\mathbb{R}^{3}} & \int_{Q} \epsilon_{1}^{-1}(y)[I+\operatorname{curl} M(y)] \operatorname{curl} u(x) \cdot \operatorname{curl}_{x} \nabla_{y} \phi^{1}(x, y) d y d x \\
& =-\int_{\mathbb{R}^{3}} \int_{Q} \epsilon_{1}^{-1}(y)[I+\operatorname{curl} M(y)] \operatorname{curl} u(x) \cdot \operatorname{curl}_{y} \nabla_{x} \phi^{1}(x, y) d y d x \\
& =-\int_{\mathbb{R}^{3}} \int_{Q} \operatorname{curl}_{y}\left(\epsilon_{1}^{-1}(y)[I+\operatorname{curl} M(y)]\right) \operatorname{curl} u(x) \cdot \nabla_{x} \phi^{1}(x, y) d y d x \\
& =0 .
\end{aligned}
$$

As was noticed in Lemma 3.1, for all $\left(\phi, \phi^{1}, \phi^{2}\right) \in\left[C_{0}^{\infty}\left(\mathbb{R}^{3}\right)\right]^{3} \times C_{0}^{\infty}\left(\mathbb{R}^{3}\right.$; $\left.C_{\#}^{\infty}(Q)\right) \times\left[C_{0}^{\infty}\left(\mathbb{R}^{3} ; C_{0}^{\infty}\left(Q_{0}\right)\right)\right]^{3}$ the function $\phi^{0}(x, y)=\phi(x)+\nabla_{y} \phi^{1}(x$, $y)+\phi^{2}(x, y)$ is an element of $C_{0}^{\infty}\left(\mathbb{R}^{3} ; V \cap\left[C_{\#}^{\infty}(Q)\right]^{3}\right)$. Equations (19), (40), (41) and (42), along with the identity $\operatorname{curl}_{y} \nabla_{y}=0$, imply that for such triplets $\left(\phi, \phi^{1}, \phi^{2}\right)$ one has

$$
\begin{aligned}
\int_{\mathbb{R}^{3}} & A^{\text {hom }} \operatorname{curl} u(x) \cdot \operatorname{curl} \phi(x) d x \\
& +\int_{\mathbb{R}^{3}} \int_{Q} \epsilon_{0}^{-1}(y) \operatorname{curl} y u^{2}(x, y) \cdot \operatorname{curl}_{y} \phi^{2}(x, y) d y d x \\
& +\int_{\mathbb{R}^{3}} \int_{Q}\left(u(x)+\nabla_{y} u^{1}(x, y)+u^{2}(x, y)\right) \\
& \times\left(\phi(x)+\nabla_{y} \phi^{1}(x, y)+\phi^{2}(x, y)\right) d y d x \\
= & \int_{\mathbb{R}^{3}} \int_{Q} f(x, y) \cdot\left(\phi(x)+\nabla_{y} \phi^{1}(x, y)+\phi^{2}(x, y)\right) d y d x .
\end{aligned}
$$

Using a closure argument, we infer that (43) holds for all $\left(\phi, \phi^{1}, \phi^{2}\right) \in \mathcal{H}$. (See Theorem 2.1 for the definition of the space $\mathcal{H}$ and note that $H_{\text {curl }}$ and $V$ contain dense subsets of smooth functions, which can be seen by arguing with mollifications as in Step 2 of the proof of Lemma 4.1.) Therefore, equation (43) is the weak formulation of the first two equations of the system (6)-(8). 
Further, we claim that if the strong two-scale convergence $f^{\eta} \stackrel{2}{\rightarrow} f$ holds, then the convergence of solutions is strong, too, i.e. $u^{\eta} \stackrel{2}{\rightarrow} u^{0}$. Since strong twoscale convergence of $f^{\eta}$ to $f$ implies weak two-scale convergence, the above result immediately implies that $u^{\eta}$ weakly two-scale converges to $u^{0}$. Therefore, it remains to show (see e.g. [2]) that

$$
\lim _{\eta \rightarrow 0} \int_{\mathbb{R}^{3}}\left|u^{\eta}(x)\right|^{2} d x=\int_{\mathbb{R}^{3}} \int_{Q}\left|u^{0}(x, y)\right|^{2} d y d x .
$$

To this end, we follow the approach of [28]. Consider the solution $w^{\eta} \in$ $\left[H^{1}\left(\mathbb{R}^{3}\right)\right]^{3}$ to the problem

$$
\begin{gathered}
\int_{\mathbb{R}^{3}}\left(\epsilon_{1}^{-1}+\eta^{2} \epsilon_{0}^{-1}\right)\left(\frac{x}{\eta}\right) \operatorname{curl} w^{\eta}(x) \cdot \operatorname{curl} \phi(x) d x+\int_{\mathbb{R}^{3}} w^{\eta}(x) \cdot \phi(x) d x \\
=\int_{\mathbb{R}^{3}} u^{\eta}(x) \cdot \phi(x) d x \quad \text { for all } \phi \in\left[H^{1}\left(\mathbb{R}^{3}\right)\right]^{3} .
\end{gathered}
$$

Using Lemma 4.1 and the present proof above, we argue that $w^{\eta}(x) \stackrel{2}{\rightarrow} w(x)+$ $\nabla_{y} w^{1}(x, y)+w^{2}(x, y)$, where the triplet $\left(w, w^{1}, w^{2}\right) \in \mathcal{H}$ satisfies the identity

$$
\begin{aligned}
\int_{\mathbb{R}^{3}} & A^{\text {hom }} \operatorname{curl} w(x) \cdot \operatorname{curl} \phi(x) d x \\
& +\int_{\mathbb{R}^{3}} \int_{Q} \epsilon_{0}^{-1}(y) \operatorname{curl} y w^{2}(x, y) \cdot \operatorname{curl}_{y} \phi^{2}(x, y) d y d x \\
& +\int_{\mathbb{R}^{3}} \int_{Q}\left(w(x)+\nabla_{y} w^{1}(x, y)+w^{2}(x, y)\right) \\
& \times\left(\phi(x)+\nabla_{y} \phi^{1}(x, y)+\phi^{2}(x, y)\right) d y d x \\
= & \int_{\mathbb{R}} \int_{Q} u^{0}(x, y) \cdot\left(\phi(x)+\nabla_{y} \phi^{1}(x, y)\right. \\
& \left.+\phi^{2}(x, y)\right) d y d x \quad \text { for all }\left(\phi, \phi^{1}, \phi^{2}\right) \in \mathcal{H} .
\end{aligned}
$$

Taking $\phi=u^{\eta}$ and $\phi=w^{\eta}$ as test functions in (45) and (25), respectively, implies that

$$
\int_{\mathbb{R}^{3}} u^{\eta} \cdot u^{\eta}=\int_{\mathbb{R}^{3}} f^{\eta} \cdot w^{\eta}
$$

which, by the strong two-scale convergence of the sequence $f^{\eta}$, gives

$$
\lim _{\eta \rightarrow 0} \int_{\mathbb{R}^{3}} u^{\eta} \cdot u^{\eta}=\int_{\mathbb{R}^{3}} \int_{Q} f^{0}(x, y) \cdot w^{0}(x, y) d y d x .
$$

Finally, taking $\left(\phi, \phi^{1}, \phi^{2}\right)=\left(w, w^{1}, w^{2}\right)$ and $\left(\phi, \phi^{1}, \phi^{2}\right)=\left(u, u^{1}, u^{2}\right)$ as test functions in (43) and (46), respectively, implies that

$$
\int_{\mathbb{R}^{3}} \int_{Q} f^{0}(x, y) \cdot w^{0}(x, y) d y d x=\int_{\mathbb{R}^{3}} \int_{Q} u^{0}(x, y) \cdot u^{0}(x, y) d y d x,
$$

which, in combination with (47), yields (44). 
4.3. Properties of the two-scale limit. We begin this section by studying the homogenized matrix $A^{\text {hom }}$ which appears in Theorem 2.1.

LEMMA 4.4. Let $A^{\text {hom }}$ denote the matrix

$$
A_{p q}^{\mathrm{hom}}=\int_{Q} \epsilon_{1}^{-1}\left(\operatorname{curl} M_{p}^{q}+\delta_{p q}\right), \quad p, q=1,2,3,
$$

where $M^{q}, q=1,2,3$, is the unique solution in $V^{\perp}$ to the problem

$$
\operatorname{curl}\left(\epsilon_{1}^{-1}\left[\operatorname{curl} M^{q}+e_{q}\right]\right)=0
$$

Then $A^{\text {hom }}$ is a positive-definite symmetric matrix. Moreover, the identity

$$
A^{\text {hom }}=\left(\epsilon_{\text {stiff }}^{\text {hom }}\right)^{-1}
$$

holds. Here $\epsilon_{\mathrm{stiff}}^{\mathrm{hom}}$ is the positive-definite symmetric matrix given by

$$
\epsilon_{\text {stiff }}^{\mathrm{hom}} \xi \cdot \xi:=\inf _{\substack{u \in H_{\#}^{1}(Q), \nabla u=-\xi \text { in } Q_{0}}} \int_{Q_{1}} \epsilon_{1}(\xi+\nabla u) \cdot(\xi+\nabla u), \quad \xi \in \mathbb{R}^{3}
$$

Proof. Suppose that $\xi \in \mathbb{R}^{3}$ and let $M_{\xi} \in V^{\perp}$ denote the unique solution, see Lemma 3.4, to

$$
\int_{Q} \epsilon_{1}^{-1}\left(\operatorname{curl} M_{\xi}+\xi\right) \cdot \operatorname{curl} w=0 \quad \text { for all } w \in V^{\perp} .
$$

We introduce the matrix $A^{\text {hom }}: \mathbb{R}^{3} \rightarrow \mathbb{R}^{3}$ as follows:

$$
A^{\text {hom }_{\xi}}:=\int_{Q} \epsilon_{1}^{-1}\left(\operatorname{curl} M_{\xi}+\xi\right) .
$$

Denoting by $M^{q}$ the vector field $M_{e_{q}}$, and taking the inner product of $A^{\text {hom }} e_{q}$ with $e_{p}$, shows that the elements of $A^{\text {hom }}$ satisfy (48). Let $\xi, \eta \in \mathbb{R}^{3}$ and let $M_{\xi}$, $M_{\eta}$ be the corresponding solutions of (49); then we readily find the identity

$$
A^{\text {hom }} \xi \cdot \eta=\int_{Q} \epsilon_{1}^{-1}\left(\operatorname{curl} M_{\xi}+\xi\right) \cdot\left(\operatorname{curl} M_{\eta}+\eta\right), \quad \xi, \eta \in \mathbb{R}^{n} .
$$

Indeed, (51) directly follows by taking the inner product on both sides of (50) with $\eta$ and setting $\psi=M_{\eta}$ in (49). Identity (51) immediately implies that $A^{\text {hom }}$ is non-negative and symmetric.

We now prove the upper bound of $A^{\text {hom }}$ by $\left(\epsilon_{\text {stiff }}^{\text {hom }}\right)^{-1}$ :

$$
A^{\text {hom }_{\xi}} \cdot \xi \leqslant\left(\epsilon_{\text {stiff }}^{\text {hom }}\right)^{-1} \xi \cdot \xi \text { for all } \xi \in \mathbb{R}^{3}
$$


First, we recall that the inverse matrix $\left(\epsilon_{\text {stiff }}^{\text {hom }}\right)^{-1}$ can be represented as follows:

$$
\left(\epsilon_{\text {stiff }}^{\text {hom }}\right)^{-1} \xi \cdot \xi:=\inf _{\substack{v \in\left[L^{2}(Q)\right]_{\text {sol }}^{3}, \int_{Q} v=0}} \int_{Q_{1}} \epsilon_{1}^{-1}(\xi+v) \cdot(\xi+v), \quad \xi \in \mathbb{R}^{3} ;
$$

see $[14$, p. 102] . Then (52) directly follows from (51) and the trivial statement that for $w \in V^{\perp}$, the function curl $w$ is an element of $\left\{v \in\left[L^{2}(Q)\right]_{\text {sol }}^{3} \mid \int_{Q} v=\right.$ $0\}$. It remains to prove the corresponding lower bound on $A^{\text {hom }}$, i.e.

$$
\left(\epsilon_{\text {stiff }}^{\text {hom }}\right)^{-1} \xi \cdot \xi \leqslant A^{\text {hom }} \xi \cdot \xi \text { for all } \xi \in \mathbb{R}^{3} .
$$

First, we will provide another representation of $A^{\text {hom }}$. Namely, we claim that

$$
A^{\operatorname{hom}} \xi \cdot \xi=\min _{M \in\left[H_{\#}^{1}(Q)\right]^{3}} \int_{Q_{1}} \epsilon_{1}^{-1}(\operatorname{curl} M+\xi) \cdot(\operatorname{curl} M+\xi), \quad \xi \in \mathbb{R}^{3} .
$$

Indeed, we see this by noting that for the functional

$$
F_{\xi}(M):=\int_{Q_{1}} \epsilon_{1}^{-1}(\operatorname{curl} M+\xi) \cdot(\operatorname{curl} M+\xi),
$$

we find $F_{\xi}(u)=F_{\xi}\left(P_{V^{\perp}} u\right)$ for all $u \in\left[H_{\#}^{1}(Q)\right]^{3}$, where $P_{V^{\perp}}$ is the orthogonal projection on to $V^{\perp}$. Therefore, $F_{\xi}$ can, without loss of generality, be minimized on $V^{\perp}$ for which (49) is the corresponding Euler-Lagrange equation.

We will now give the proof of (53). Let us recall that for an invertible matrix $A: \mathbb{R}^{3} \rightarrow \mathbb{R}^{3}$, the solution to the linear system $A x=b$ is also the minimum of the functional $F(\eta):=(A \eta \cdot \eta) / 2-b \cdot \eta, \eta \in \mathbb{R}^{n}$. Choosing $A$ to be the multiplication by $\epsilon_{1}$ and $b=\operatorname{curl} M(x)+\xi$, one has, for almost every $x \in \mathbb{R}^{3}$, the equality

$$
\frac{1}{2} \epsilon_{1}^{-1}(\operatorname{curl} M(x)+\xi) \cdot(\operatorname{curl} M(x)+\xi)=\sup _{\eta \in \mathbb{R}^{3}}\left\{(\operatorname{curl} M(x)+\xi) \cdot \eta-\frac{1}{2} \epsilon_{1} \eta \cdot \eta\right\} .
$$

In particular, one finds that

$$
\begin{aligned}
\frac{1}{2} A^{\text {hom } \xi \cdot \xi} & =\min _{M \in\left[H_{\#}^{1}(Q)\right]^{3}} \frac{1}{2} \int_{Q_{1}} \epsilon_{1}^{-1}(\operatorname{curl} M+\xi) \cdot(\operatorname{curl} M+\xi) \\
& =\min _{M \in\left[H_{\#}^{1}(Q)\right]^{3}} \int_{Q_{1}} \sup _{\eta \in \mathbb{R}^{3}}\left\{(\operatorname{curl} M+\xi) \cdot \eta-\frac{1}{2} \epsilon_{1} \eta \cdot \eta\right\} \\
& \geqslant \min _{M \in\left[H_{\#}^{1}(Q)\right]^{3}} \sup _{\eta \in\left[C_{0}^{\infty}(Q)\right]^{3}} \int_{Q_{1}}\left\{(\operatorname{curl} M+\xi) \cdot \eta-\frac{1}{2} \epsilon_{1} \eta \cdot \eta\right\} \\
& =\min _{M \in\left[H_{\#}^{1}(Q)\right]^{3}} \sup _{\eta \in\left[L^{2}(Q)\right]^{3}} \int_{Q_{1}}\left\{(\operatorname{curl} M+\xi) \cdot \eta-\frac{1}{2} \epsilon_{1} \eta \cdot \eta\right\} \\
& \geqslant \sup _{\eta \in\left[L^{2}(Q)\right]^{3}} \inf _{M \in\left[H_{\#}^{1}(Q)\right]^{3}} \int_{Q_{1}}\left\{(\operatorname{curl} M+\xi) \cdot \eta-\frac{1}{2} \epsilon_{1} \eta \cdot \eta\right\} .
\end{aligned}
$$


By considering the space $\mathcal{V}:=\left\{\eta \in\left[H_{\#}^{1}(Q)\right]^{3} \mid \int_{Q_{1}} \eta \cdot \operatorname{curl} \psi\right.$ for all $\psi \in$ $\left.\left[H_{\#}^{1}(Q)\right]^{3}\right\}$, we then infer that

$$
\begin{aligned}
\frac{1}{2} A^{\operatorname{hom}} \xi \cdot \xi & \geqslant \sup _{\eta \in \mathcal{V}} \inf _{M \in\left[H_{\#}^{1}(Q)\right]^{3}} \int_{Q_{1}}\left\{(\operatorname{curl} M+\xi) \cdot \eta-\frac{1}{2} \epsilon_{1} \eta \cdot \eta\right\} \\
& =\sup _{\eta \in \mathcal{V}} \inf _{M \in\left[H_{\#}^{1}(Q)\right]^{3}} \int_{Q_{1}}\left\{\xi \cdot \eta-\frac{1}{2} \epsilon_{1} \eta \cdot \eta\right\} \\
& =\sup _{\eta \in \mathcal{V}} \int_{Q_{1}}\left\{\xi \cdot \eta-\frac{1}{2} \epsilon_{1} \eta \cdot \eta\right\} .
\end{aligned}
$$

Now, we note that the space $\left\{a+\nabla \varphi \mid a \in \mathbb{R}^{3}, \varphi \in\left[H_{\#}^{1}(Q)\right]^{3}, \nabla \varphi=-a\right.$ in $\left.Q_{0}\right\}$ is a subset of $\mathcal{V}$ and therefore

$$
\begin{aligned}
\frac{1}{2} A^{\text {hom }} \xi \cdot \xi \geqslant & \sup _{\substack{\eta=a+\nabla \varphi, a \in \mathbb{R}^{3}, \varphi \in H_{\#}^{1}\left(Q_{1}\right), \nabla \varphi=-a \text { in } Q_{0}}} \int_{Q}\left\{\xi \cdot \eta-\frac{1}{2} \epsilon_{1} \eta \cdot \eta\right\} \\
& =-\inf _{a \in \mathbb{R}^{3}} \inf _{\substack{\varphi \in H_{\#}^{1}\left(Q_{1}\right), \nabla \varphi=-a \text { in } Q_{0}}} \int_{Q}\left\{\frac{1}{2} \epsilon_{1}(a+\nabla \varphi) \cdot(a+\nabla \varphi)-\xi \cdot(a+\nabla \varphi)\right\} \\
& =-\inf _{a \in \mathbb{R}^{3}} \inf _{\substack{\varphi \in H_{\#}^{1}\left(Q_{1}\right), \nabla \varphi=-a \text { in } Q_{0}}}\left\{\frac{1}{2} \int_{Q} \epsilon_{1}(a+\nabla \varphi) \cdot(a+\nabla \varphi)-\xi \cdot a\right\} \\
& =-\inf _{a \in \mathbb{R}^{3}}\left\{\frac{1}{2} \epsilon_{\text {htiff }}^{\text {stiff }} a \cdot a-\xi \cdot a\right\} .
\end{aligned}
$$

(Here $\left[H_{\#}^{1}\left(Q_{1}\right)\right]^{3}$ consists of restrictions to $Q_{1}$ of elements of $\left[H_{\#}^{1}(Q)\right]^{3}$.) Arguing as in (54), we arrive at (53), i.e.

$$
\frac{1}{2} A^{\mathrm{hom}} \xi \cdot \xi \geqslant \frac{1}{2}\left(\epsilon_{\mathrm{hom}}^{\mathrm{stiff}}\right)^{-1} \xi \cdot \xi, \quad \xi \in \mathbb{R}^{3} .
$$

To conclude this section. we provide an equivalent formulation for the system (6)-(8) and describe its solution set.

Definition 4.5. Suppose that $f \in\left[L^{2}\left(\mathbb{R}^{3} \times Q\right)\right]^{3}, \operatorname{div}_{y} f(x, y)=0$ and consider solutions to the system (55)-(59):

$$
\begin{aligned}
& \operatorname{curl}_{x}\left(A^{\text {hom }} \operatorname{curl}_{x} u(x)\right)+u(x)+\int_{Q_{0}} z(x, y) d y+\int_{Q_{1}} \nabla_{y} v(x, y) d y \\
&= \int_{Q} f(x, y) d y \\
& \operatorname{curl}_{y}\left(\epsilon_{0}^{-1}(y) \operatorname{curl}_{y} z(x, y)\right)+z(x, y)=f(x, y)-u(x) \text { in } Q_{0},
\end{aligned}
$$




$$
\begin{gathered}
\operatorname{div}_{y} z(x, y)=0 \text { in } Q_{0}, \\
\Delta_{y} v(x, y)=0 \text { in } Q_{1}, \\
z(x, y)=\nabla_{y} v(x, y) \text { on } \partial Q_{0} .
\end{gathered}
$$

The solution to the above problem is understood in the following sense. Consider the bilinear form

$$
\begin{aligned}
\mathfrak{B}(u, \phi)= & \int_{\mathbb{R}^{3}} A^{\text {hom }} \operatorname{curl} a(u)(x) \cdot \operatorname{curl} a(\phi)(x) d x \\
& +\int_{\mathbb{R}^{3}} \int_{Q_{0}} \epsilon_{0}^{-1} \operatorname{curl}_{y} u \cdot \operatorname{curl}_{y} \phi d y d x \\
& +\int_{\mathbb{R}^{3}} \int_{Q} u \cdot \phi d y d x, \quad u, \phi \in \mathcal{U},
\end{aligned}
$$

where

$$
\begin{aligned}
\mathcal{U}=\left\{u \in\left[L^{2}\left(\mathbb{R}^{3} ; H_{\#}^{1}(Q)\right)\right]^{3}:\left.u\right|_{y \in Q_{1}}=a(u)+\nabla_{y} p_{u},\right. \\
\left.p_{u} \in L^{2}\left(\mathbb{R}^{3} ; H_{\#}^{2}(Q)\right), a(u) \in H_{\mathrm{curl}}, \operatorname{div}_{y} u=0\right\}
\end{aligned}
$$

and $a(v)$ is defined by Lemma 3.1 for elements $v$ of $\in \mathcal{U}$ considered as functions of the variable $y \in Q$. We say that $(u, v, z) \in H_{\text {curl }} \times\left[L^{2}\left(\mathbb{R}^{3} ; H_{\#}^{2}(Q)\right)\right]^{3} \times$ $\left[L^{2}\left(\mathbb{R}^{3} ; H^{1}\left(Q_{0}\right)\right)\right]^{3}$ is a solution to (55)-(59) if there exists $\hat{u} \in \mathcal{U}$ such that $u=a(\hat{u}), v=p_{\hat{u}}, z=\hat{u}-a(\hat{u})$ and

$$
\mathfrak{B}(\hat{u}, \phi)=\int_{Q} f \cdot \phi \quad \text { for all } \phi \in \mathcal{U} .
$$

Remark 4.6. The equation (55) of the above system is formally equivalent to setting $\phi=\varphi$, where $\varphi \in\left[C_{0}^{\infty}\left(\mathbb{R}^{3}\right)\right]^{3}$ in (60). This can be seen by noticing that $\int_{Q} u(x) d y=u(x)$ and that $\int_{Q_{0}} z(x, y) d y, \int_{Q_{1}} \nabla_{y} v(x, y) d y$ are elements of $\left[L^{2}\left(\mathbb{R}^{3}\right)\right]^{3}$ as functions of $x$. The equations (56)-(59) are formally equivalent to taking $\phi$ of the form $\phi(x, y)=\varphi(x) \rho(y)$, where $\varphi \in C_{0}^{\infty}\left(\mathbb{R}^{3}\right)$ and $\rho \in$ $\left[C_{\#}^{\infty}(Q)\right]^{3}$ is such that $\left.\rho\right|_{Q_{1}}=\nabla \omega, \omega \in C_{\#}^{\infty}(Q)$ (i.e. $a(\rho)=0$ ) and $\operatorname{div} \rho=0$.

Remark 4.7. Notice that the interface condition (59) is equivalent to the pair of conditions

$$
z \times\left. n\right|_{\partial Q_{0}}=\nabla v \times\left. n\right|_{\partial Q_{0}},\left.\quad \frac{\partial v}{\partial n}\right|_{\partial Q_{0}}=\left.z \cdot n\right|_{\partial Q_{0}},
$$

where $n$ is a unit normal to $\partial Q_{0}$.

Proposition 4.8. Suppose that $f \in\left[L^{2}\left(\mathbb{R}^{3} \times Q\right)\right]^{3}$ is such that $\operatorname{div}_{y} f(x$, $y)=0$; then the problem (60) has a unique solution $\hat{u} \in \mathcal{U}$. For the solution $\hat{u}$ of (60), the triplet $\left(a(\hat{u}), p_{\hat{u}}, \hat{u}-a(\hat{u})-\nabla_{y} p_{\hat{u}}\right)$ is a solution to (7)-(8). Conversely, for any solution $(u, v, z)$ to the system (6)-(8), the function $\hat{u}=u+\nabla_{y} v+z$, belongs to $\mathcal{U}$ and is a solution to (60); in other words, $\left(u, v, \nabla_{y} v+z\right)$ is a solution to (55)-(59). 
Corollary 4.9. Suppose that $f \in\left[L^{2}\left(\mathbb{R}^{3} \times Q\right)\right]^{3}$ is such that $\operatorname{div}_{y} f(x$, $y)=0$; then the system (7)-(8) has a solution. For any two solutions $\left(u_{1}, v_{1}, z_{1}\right)$, $\left(u_{2}, v_{2}, z_{2}\right)$ to the system (7)-(8), one has $u_{1}=u_{2}, \nabla v_{1}-\nabla v_{2}=z_{2}-z_{1}=\nabla \hat{\psi}$ for some function $\hat{\psi} \in H_{\#}^{2}(Q) \cap H_{0}^{1}\left(Q_{0}\right)$.

Proof. Suppose that $f \in\left[L^{2}\left(\mathbb{R}^{3} \times Q\right)\right]^{3}$ is such that $\operatorname{div}_{y} f(x, y)=0$ and let $\left(u_{1}, v_{1}, z_{1}\right),\left(u_{2}, v_{2}, z_{2}\right) \in H_{\text {curl }} \times L^{2}\left(\mathbb{R}^{3} ; H_{\#}^{2}(Q)\right) \times\left[L^{2}\left(\mathbb{R}^{3} ; H_{0}^{1}\left(Q_{0}\right)\right)\right]^{3}$ be two solutions to the system (6)-(8). Then, by Proposition 4.8, $u_{1}+\nabla_{y} v_{1}+z_{1}$ and $u_{2}+\nabla_{y} v_{2}+z_{2}$ are solutions to (60). In particular, by the uniqueness of solutions to (60), we find that $u_{1}+\nabla_{y} v_{1}+z_{1}=u_{2}+\nabla_{y} v_{2}+z_{2}$. Furthermore, by Lemma 3.1, one has

$\left\|u_{1}-u_{2}\right\|_{\left[L^{2}\left(\mathbb{R}^{3}\right)\right]^{3}} \leqslant c\left\|\left(u_{1}+\nabla_{y} v_{1}+z_{1}\right)-\left(u_{2}+\nabla_{y} v_{2}+z_{2}\right)\right\|_{\left[L^{2}\left(\mathbb{R}^{3} ; H_{\#}^{1}(Q)\right)\right]^{3}}=0$.

Proof of Proposition 4.8. We endow the vector space $\mathcal{U}$ with the inner product

$$
\begin{aligned}
(u, \phi) \mathcal{U}= & \int_{\mathbb{R}^{3}} \operatorname{curl} a(u)(x) \cdot \operatorname{curl} a(\phi)(x) d x \\
& +\int_{\mathbb{R}^{3}} \int_{Q_{0}} \operatorname{curl}_{y} u(x, y) \cdot \operatorname{curl}_{y} \phi(x, y) d y d x \\
& +\int_{\mathbb{R}^{3}} \int_{Q} u(x, y) \cdot \phi(x, y) d y d x, \quad u, \phi \in \mathcal{U},
\end{aligned}
$$

with the induced norm

$$
\begin{aligned}
\|u\|_{\mathcal{U}}^{2}= & \int_{\mathbb{R}^{3}}|\operatorname{curl} a(u)(x)|^{2} d x+\int_{\mathbb{R}^{3}} \int_{Q_{0}}\left|\operatorname{curl}_{y} u(x, y)\right|^{2} d y d x \\
& +\int_{\mathbb{R}^{3}} \int_{Q}|u(x, y)|^{2} d y d x, \quad u \in \mathcal{U} .
\end{aligned}
$$

We will now show that $\mathcal{U}$ is a Hilbert space. For a given Cauchy sequence $u_{n} \in$ $\mathcal{U}$, we observe that $u_{n}$ is Cauchy in $\left[L^{2}\left(\mathbb{R}^{3} ; H_{\#}^{1}(Q)\right)\right]^{3}$ since

$$
\int_{\mathbb{R}^{3}} \int_{Q_{0}}\left|\operatorname{curl}_{y} u_{n}(x, y)\right|^{2} d y d x+\int_{\mathbb{R}^{3}} \int_{Q}\left|u_{n}(x, y)\right|^{2} d y d x=\left\|u_{n}\right\|_{L^{2}\left(\mathbb{R}^{3} ; H_{\#}^{1}(Q)\right)}^{2} .
$$

Therefore, $u_{n}$ converges strongly to an element $u \in\left[L^{2}\left(\mathbb{R}^{3} ; H_{\#}^{1}(Q)\right)\right]^{3}$. The condition $\operatorname{div}_{y} u_{n}=0$ implies that $\operatorname{div}_{y} u=0$. Moreover, as $u_{n} \in L^{2}\left(\mathbb{R}^{3} ; V\right)$ (see (9)), $u \in L^{2}\left(\mathbb{R}^{3} ; V\right)$ and, by Lemma 3.1, $u=a+\nabla_{y} b$ in $Q_{1}$ for some $a \in$ $\left[L^{2}\left(\mathbb{R}^{3}\right)\right]^{3}$ and $b \in L^{2}\left(\mathbb{R}^{3} ; H_{\#}^{2}(Q)\right)$. It remains to show that curl $a \in\left[L^{2}\left(\mathbb{R}^{3} \times\right.\right.$ $Q)]^{3}$. Note that, by (61), curl $a\left(u_{n}\right)$ is a Cauchy sequence in $\left[L^{2}\left(\mathbb{R}^{3} \times Q\right)\right]^{2}$, which implies that it has a strong limit $c \in\left[L^{2}\left(\mathbb{R}^{3} \times Q\right)\right]^{2}$. Let us now show that $c=\operatorname{curl} a$. For a fixed $\phi \in\left[C_{0}^{\infty}\left(\mathbb{R}^{3}\right)\right]^{3}$, let $\Psi_{\phi} \in W \cap\left[C^{\infty}\left(\mathbb{R}^{3} ; L^{2}(Q)\right)\right]^{3}$ be 
given by Proposition 4.3. Then, upon recalling (35) and (31), we find that

$$
\begin{aligned}
\int_{\mathbb{R}^{3}} & \operatorname{curl} a\left(u_{n}\right)(x) \cdot \phi(x) d x \\
& =\int_{\mathbb{R}^{3}} a\left(u_{n}\right)(x) \cdot \operatorname{curl} \phi(x) d x \\
& =\int_{\mathbb{R}^{3}} \int_{Q} a\left(u_{n}\right)(x) \cdot \operatorname{curl}_{x}\left(\epsilon_{1}^{-1 / 2}(y) \Psi_{\phi}(x, y)\right) d y d x \\
& =\int_{\mathbb{R}^{3}} \int_{Q}\left(a\left(u_{n}\right)(x)+\nabla_{y} p_{u_{n}}(x, y)\right) \cdot \operatorname{curl}_{x}\left(\epsilon_{1}^{-1 / 2}(y) \Psi_{\phi}(x, y)\right) d y d x \\
& =\int_{\mathbb{R}^{3}} \int_{Q} u_{n}(x, y) \cdot \operatorname{curl}_{x}\left(\epsilon_{1}^{-1 / 2}(y) \Psi_{\phi}(x, y)\right) d y d x
\end{aligned}
$$

Passing to the limit $n \rightarrow \infty$ in (62) gives

$$
\begin{aligned}
\int_{\mathbb{R}^{3}} c(x) \cdot \phi(x) d x \\
\quad=\int_{\mathbb{R}^{3}} \int_{Q} u(x, y) \cdot \operatorname{curl}_{x}\left(\epsilon_{1}^{-1 / 2}(y) \Psi_{\phi}(x, y)\right) d y d x \\
\quad=\int_{\mathbb{R}^{3}} \int_{Q}\left(a(x)+\nabla_{y} b(x, y)\right) \cdot \operatorname{curl}_{x}\left(\epsilon_{1}^{-1 / 2}(y) \Psi_{\phi}(x, y)\right) d y d x \\
=\int_{\mathbb{R}^{3}} \int_{Q} a(x) \cdot \operatorname{curl}_{x}\left(\epsilon_{1}^{-1 / 2}(y) \Psi_{\phi}(x, y)\right) d y d x,
\end{aligned}
$$

which, by another application of (35), implies that

$$
\int_{\mathbb{R}^{3}} c(x) \cdot \phi(x) d x=\int_{\mathbb{R}^{3}} a(x) \cdot \operatorname{curl} \phi(x) d x \quad \text { for all } \phi \in\left[C_{0}^{\infty}\left(\mathbb{R}^{3}\right)\right]^{3} .
$$

Hence, $\mathcal{U}$ is complete.

Now that we have shown that $\mathcal{U}$ is a Hilbert space, the existence and uniqueness to problem (60) follow by noting that

$$
\begin{aligned}
\mathfrak{B}(u, \phi)= & \int_{\mathbb{R}^{3}} A^{\text {hom }} \operatorname{curl} a(u) \cdot \operatorname{curl} a(\phi) \\
& +\int_{\mathbb{R}^{3}} \int_{Q_{0}} \epsilon_{0}^{-1} \operatorname{curl}_{y} u \cdot \operatorname{curl}_{y} \phi+\int_{\mathbb{R}^{3}} \int_{Q} u \cdot \phi
\end{aligned}
$$

is an equivalent inner product on $\mathcal{U}$ and invoking the Riesz representation theorem. Indeed, the equivalence follows directly from (61) and recalling that $A^{\text {hom }}$ is positive definite and that $\epsilon_{0}^{-1} \in L^{\infty}(Q)$ is positive in $Q_{0}$.

The remaining part of the statement follows by a direct check of equivalence of (6)-(8) and (55)-(59) under the condition $\hat{u}=u+\nabla_{y} u^{1}+u^{2}$. 
Acknowledgements. This work was carried out under the financial support of the Leverhulme Trust (Grant RPG-167 "Dissipative and non-self-adjoint problems") and the Engineering and Physical Sciences Research Council (Grant EP/L018802/1 "Mathematical foundations of metamaterials: homogenisation, dissipation and operator theory").

\section{References}

1. R. A. Adams, Sobolev Spaces, Academic Press (New York-San Francisco-London, 1975).

2. G. Allaire, Homogenization and two-scale convergence. SIAM J. Math. Anal. 23 (1992), 1482-1518.

3. A. Bensoussan, J.-L. Lions and G. C. Papanicolaou, Asymptotic Analysis for Periodic Structures, North-Holland (1978).

4. G. Berkolaiko and P. Kuchment, Introduction to Quantum Graphs (Mathematical Surveys and Monographs 186), American Mathematical Society (Providence, RI, 2013).

5. K. D. Cherednichenko, Two-scale asymptotics for non-local effects in composites with highly anisotropic fibres. Asymptot. Anal. 49 (2006), 39-59.

6. K. D. Cherednichenko and S. Cooper, Resolvent estimates for high-contrast elliptic problems with periodic coefficients, submitted (2013).

7. K. D. Cherednichenko, S. Cooper and S. Guenneau, Spectral analysis of one-dimensional highcontrast elliptic problems with periodic coefficients. Multiscale Model. Simul. 13 (2015), 72-98.

8. D. Cioranescu, A. Damlamian and G. Griso, Periodic unfolding and homogenisation. C. R. Math. Acad. Sci. Paris 335(1) (2002), 99-104.

9. S. Cooper, Two-scale homogenisation of partially degenerating PDEs with applications to photonic crystals and elasticity. PhD Thesis, University of Bath, 2012.

10. R. Dautray and J.-L. Lions, Mathematical Analysis and Numerical Methods for Science and Technology, Vol. 3 (Spectral Theory and Applications), Springer (Berlin, 1990).

11. L. Evans, Partial Differential Equations (Graduate Studies in Mathematics 19), American Mathematical Society (Providence, RI, 2010).

12. A. Figotin and P. Kuchment, Spectral properties of classical waves in high-contrast periodic media. SIAM J. Appl. Math. 58 (1998), 683-702.

13. R. Hempel and K. Lienau, Spectral properties of periodic media in the large coupling limit. Comm. Partial Differential Equations 25 (2000), 1445-1470.

14. V. V. Jikov, S. M. Kozlov and O. A. Oleinik, Homogenization of Differential Operators and Integral Functionals, Springer (1994).

15. J. D. Joannopoulos, R. D. Meade and J. N. Winn, Photonic Crystals: Molding the Flow of Light, Princeton University Press (Princeton, NJ, 1995).

16. I. V. Kamotski and V. P. Smyshlyaev, 2006, Localised modes due to defects in high contrast periodic media via homogenisation. BICS preprint 3/06, 2006, available online at: http://www.bath.ac.uk/ mathsci/bics/preprints/bics063.pdf.

17. I. V. Kamotski and V. P. Smyshlyaev, Two-scale homogenization for a class of partially degenerating PDE systems. Preprint, 2013, arXiv:1309.4579v1.

18. V. A. Kozlov, V. G. Maz'ya and A. B. Movchan, Asymptotic Analysis of Fields in Multi-Structures, Oxford University Press (Oxford, 1999).

19. P. Kuchment, Floquet Theory for Partial Differential Equations (Operator Theory: Advances and Applications 60), Birkhäuser (Basel, 1993).

20. V. G. Maz'ya, Sobolev Spaces, Springer (Berlin-Tokyo, 1985).

21. V. G. Maz'ya, S. A. Nazarov and B. A. Plamenevskii, Asymptotic Theory of Elliptic Boundary Value Problems in Singularly Perturbed Domains, Vol. II (Operator Theory: Advances and Applications 112), Birkhäuser (Basel, 2000).

22. G. Nguetseng, A general convergence result for a functional related to the theory of homogenization. SIAM J. Math. Anal. 20(3) (1989), 608-623.

23. L. Parnovski, Bethe-Sommerfeld conjecture. Ann. Henri Poincaré 9(3) (2008), 457-508.

24. S. E. Pastukhova, Asymptotic analysis of elasticity problems on thin periodic structures. Netw. Heterog. Media 4(3) (2009), 577-604.

25. P. Russell, Photonic crystal fibers. Science 299 (2003), 358-362. 
26. G. V. Sandrakov, Averaging of non-stationary equations with contrast coefficients. Dokl. Akad. Nauk 355(5) (1997), 605-608.

27. E. M. Stein, Singular Integrals and Differentiability Properties of Functions, Princeton University Press (Princeton, NJ, 1970).

28. V. V. Zhikov, On an extension of the method of two-scale convergence and its applications. Sb. Math. 191(7) (2000), 973-1014.

29. V. V. Zhikov, On gaps in the spectrum of some divergent elliptic operators with periodic coefficients. St. Petersburg Math. J. 16(5) (2004), 719-773.

Kirill Cherednichenko,

Department of Mathematical Sciences,

University of Bath,

Claverton Down,

Bath BA2 7AY,

U.K.

E-mail: K.Cherednichenko@bath.ac.uk
Shane Cooper,

Laboratoire de Mécanique et Génie Civil de Montpellier,

860 Rue de Saint-Priest, 34095 Montpellier,

France

E-mail: salcoops@gmail.com 